\title{
Nonparametric estimation of structural models for high-frequency currency market data
}

\author{
Ravi Bansal ${ }^{a}$, A. Ronald Gallant ${ }^{b}$, Robert Hussey ${ }^{c}$, George Tauchen*a \\ a Duke University, Durham, NC 27706, USA \\ ${ }^{\mathrm{b}}$ University of North Carolina, Chapel Hill, NC 27599, USA \\ ' Georgetown University, Washington, DC 20007, USA
}

(Received July 1992; final version received January 1994)

\begin{abstract}
Empirical modeling of high-frequency currency market data reveals substantial evidence for nonnormality, stochastic volatility, and other nonlinearities. This paper investigates whether an equilibrium monetary model can account for nonlinearities in weekly data. The model incorporates time-nonseparable preferences and a transaction cost technology. Simulated sample paths are generated using Marcet's parameterized expectations procedure. The paper also develops a new method for estimation of structural economic models. The method forces the model to match (under a GMM criterion) the score function of a nonparametric estimate of the conditional density of observed data. The estimation uses weekly U.S.-German currency market data, 1975-90.
\end{abstract}

Key words: Monetary model; Calibration; Simulation estimator; Exchange rates; Nonparametric

JEL classification: $\mathrm{C} 51$

\section{Introduction}

Time series analysis provides considerable evidence that high-frequency currency market data exhibit interesting nonlinear dynamics. Documented

* Corresponding author.

This material is based upon work supported by the National Science Foundation under Grants No. SES-9111867 and SES-9023083. We thank Geert Bekaert, Lars Hansen, David Hsieh, Ellen McGrattan, Tom Sargent, and many seminar and conference participants for helpful comments at various stages of this research. 
nonlinearities include complicated patterns of time-varying nonnormality and conditional heteroskedasticity. These findings emerge from extensive examinations of short-term exchange rate movements using ARCH and GARCH methods (Bollerslev, Chou, and Kroner, 1992), nonlinear dynamic methods (Hsieh, 1989), and nonparametric time series methods (Gallant, Hsieh, and Tauchen, 1991; Engle and Gonzales-Rivera, 1991).

Besides this time series work, a series of attempts has also been made to construct parametric models that impose tight restrictions on currency market data. These parametric models have generally been rejected. At the monthly frequency, various versions of the elementary monetary (portfolio balance) model have been rejected in regression analysis (Frankel, 1984; Huang, 1981) and in out-of-sample forecasts (Meese and Rogoff, 1983a, b).

Rejections of the portfolio balance model have led some economists to argue that exchange rates are excessively variable and primarily driven by factors other than market fundamentals, such as sunspots (Dornbusch and Frankel, 1987). On the contentious issue of efficiency, one common view is that exchange markets provide little evidence of being 'efficient' (Tobin, 1978; Dooley and Shafer, 1983; Bilson 1981; Frankel and Froot, 1989). Authors who hold this view interpret the failure of the forward discount to be an unbiased predictor of expected depreciation and the biases in forecast surveys as evidence against market efficiency and stable speculation.

An alternate view, the so-called equilibrium view, taken by Singleton (1987), Stockman (1980), and others is to argue that exchange rate movements are outcomes of agents solving intertemporal optimization problems under uncertainty. Stockman (1980), Lucas (1982), Svensson (1985), Bansal (1990), Bekaert (1991), and Hodrick (1989) construct models in which prices of assets (foreign exchange rates, forward contract prices, etc.) are dependent on preferences and uncertainty in the evolution of macroeconomic fundamentals. The forward price fails to be an unbiased predictor of the expected spot price because of risk premia that emerge endogenously.

Equilibrium models are inherently nonlinear. It is thus natural to ask whether an equilibrium model can account for the rich set of nonlinearities that the time series analysis uncovers in high-frequency currency market data. To date, there is extensive literature on fitting equilibrium models to monthly and quarterly data. This line of research follows on the seminal work of Hansen and Singleton (1982). It has met with some empirical success (Hansen and Jagannathan, 1991; Cochrane and Hansen, 1992) but has never fully confronted the complicated nonlinear structure of financial data because much of the complicated nonlinearity in financial market data is lost in passing from daily or weekly frequencies to monthly or lower frequencies due to the smoothing effects of temporal aggregation (Bollerslev, Chou, and Kroner, 1992).

The primary objective of this paper is to develop an estimation strategy for implementing a nonlinear equilibrium model of exchange rates and interest rates at the weekly frequency. A complicating factor is that reliable data on consumption, 
endowments, and the money supply are not available weekly. Hence, Euler equation methods are not suitable because observations on these variables would be needed to form the Euler equation error. On the other hand, excellent data on asset returns (payoffs) are available weekly, so our estimation strategy focuses on using these data. The strategy entails making a complete specification of the model, including the law of motion of the latent driving processes, so that simulated realizations can be generated given candidate parameter settings. The criterion of fit involves comparing the time series properties of simulated versus observed realizations on exchanges rates and interest rates. With the fit criterion based on full nonparametric estimation, the approach forces the equilibrium model to confront all relevant nonlinearities present in the data.

The equilibrium model is a nonlinear, rational expectations model that generates exchange rates and interest rates endogenously via a nonlinear mapping from exogenous state variables. In the basic specification of the economy, the exogenous state processes are linear Gaussian processes; hence, all nonlinearities arise from the specification of preferences and the manner in which money enters the economy. This general setting subsumes a rich set of economies by allowing time nonseparabilities in preferences (Dunn and Singleton, 1986; Gallant and Tauchen, 1989; Sundaresan, 1989; Hansen and Jagannathan, 1991; Constantinides, 1990) and monetary shocks via a transaction costs technology (Bansal, 1990). Taking the week as the appropriate interval of decision making is important. As noted, it forces the model economy to confront the interesting nonlinear dynamics observed in the payoff data at this interval. Furthermore, a weekly decision interval seems is a more reasonable description of the behavior of economic agents than a monthly interval, especially in monetary economies.

Generating endogenous simulated time series from the economic model requires having a solution procedure for nonlinear rational expectations models. Recently several techniques have been developed to solve nonlinear models (for example, Marcet, 1991; Tauchen and Hussey, 1991). Taylor and Uhlig (1990) provide a survey of this literature and a discussion of the JBES symposium on solving nonlinear rational expectations models (Tauchen, 1990). In solving the model for this paper, we use the procedure of parameterized expectations as developed in Marcet (1991), which is well adapted for our approach.

The estimation strategy is a nonparametric version of the parametric estimator derived in Gallant and Tauchen (1992b). The estimator is based on minimizing a criterion function that contains all of the indicators (scoring functions) from a nonparametric estimate of the conditional density of the observed data. The statistical model that defines the scores is the 'score generator'. Gallant and Tauchen (1992b) examine the asymptotic theory for the case when the score generator model is a finitedimensional parametric model. Related papers that use a parametric model to define the criterion are Gourieroux, Monfort, and Renault (1992) and Smith $(1990,1992)$. In this paper, the score generator is defined by a nonparametric (sieve) estimator of the conditional density of the observed data. In one sense, made more precise 
below, the estimator is a time series analogue of the closest empirical distribution estimator of Manski (1983).

We use three series: the growth rate of the \$/DM exchange rate, the 30-day forward premium on this exchange rate, and a one-month treasury bill interest rate, all observed weekly.

The first step of the estimation is to use the SNP method of Gallant and Tauchen $(1989,1992 \mathrm{a})$ to estimate the conditional density of the exchange rate and interest rate data. The conditional density subsumes all the nonlinear dynamics in the data. It therefore provides a comprehensive benchmark that the model economy must reproduce, if it is to be judged a success. The fitted conditional density reveals some important features of the data that are related to particular economic issues. First, while exchange rate changes are uncorrelated, yields on nominal risk-free bonds are highly persistent. From the perspective of economic theory, it is a challenge to reconcile this difference in the temporal behavior of payoffs. Second, the densities of both the exchange rate and the interest rate display considerable leptokurtosis and skewness. Third, there is persistent stochastic volatility along with evidence for other higher-order forms of nonlinearity.

The second step is to perform GMM with the score function from the first step used to define the criterion. In effect, the parameters of the economic model are estimated in such a way that a nonparametric fit of the simulated data matches as closely as possible (under a GMM criterion) the nonparametric fit to the observed data.

The equilibrium model must therefore accommodate several aspects of the data at once. For example, the unbiasedness hypothesis for the forward rate can be rejected with our weekly data (also see Hansen and Hodrick, 1983; Hodrick and Srivastava, 1986; Cumby and Obstfeld, 1984; Baillie, Lippens, and McMahon, 1983). Thus the model economy has to be capable of producing sufficient risk premia to be a viable explanation for the observed rejection of the efficiency paradigm. In the related context of equity premia, it has been shown that different statistical procedures can lead to different implications about the risk premium (Hansen and Jagannathan, 1991; Gallant, Hansen, and Tauchen, 1990; Gallant and Tauchen, 1989; Constantinides, 1990). Our approach estimates the model on a fine time interval and not merely by matching some particular moments chosen by the researcher. The latter of these two issues is relevant because it is possible that a model structure that does a good job in matching some chosen moments may do miserably in other dimensions (for example, see Gallant, Hansen, and Tauchen, 1990). By using weekly data and by making the conditional density the 'moment restriction' that the model economy has to match, we directly address both these issues.

Recent papers that follow a simulated method of moment estimation strategy typically focus on some preselected moments to evaluate the model under consideration [for example, Duffie and Singleton, 1993; Bekaert, 1991; Heaton, 1993). Such a procedure has the potential for overlooking interesting aspects of the data that time series analysis reveals important, and the model should thus confront. Furthermore, 
in contrast to current practice with simulation estimators, our procedure estimates the law of motion of the forcing exogenous processes jointly with the structural economic parameters, instead of leaving the law of motion fixed at some preliminary estimate.

The remainder of the paper proceeds as follows. Section 2 lays down the structure of the assumed economy. Section 3 describes the estimation procedure. Section 4 describes the data set and the conditional density estimation. Section 5 contains our findings using the nonparametric structural estimator. Section 6 contains conclusions.

\section{The structural model}

\subsection{Preferences}

A representative world consumer has preferences defined over services from two consumption goods. The utility function is assumed to have the form

$$
\mathrm{E}_{0} \sum_{t=0}^{\infty} \beta^{t}\left[\left(c_{1 t}^{* \delta} c_{2 t}^{* 1-\delta}\right)^{1-\gamma}-1\right] /(1-\gamma),
$$

where $0<\beta<1,0<\delta<1, \gamma>0$, and where $c_{1 t}^{*}$ and $c_{2 t}^{*}$ are the consumption services from goods produced in countries 1 and 2, respectively. Preferences are of the constant relative risk aversion type in terms of the composite consumption good. The parameter $\gamma$ is the coefficient of relative risk aversion, $\delta$ determines the allocation of expenditure between the two services, and $\beta$ is the subjective discount factor. If $\gamma=1$, then preferences collapse to log-utility:

$$
\mathrm{E}_{0} \sum_{t=0}^{\infty} \beta^{t}\left[\delta \log c_{1 t}^{*}+(1-\delta) \log c_{2 t}^{*}\right] .
$$

The transformation of goods to services, as in Dunn and Singleton (1986) and Eichenbaum and Hansen (1990), is a linear technology:

$$
\begin{aligned}
& c_{1 t}^{*}=c_{1 t}+\kappa_{11} c_{1, t-1}+\cdots+\kappa_{1 L_{i}} c_{1, t-L_{i}}, \\
& c_{2 t}^{*}=c_{2 t}+\kappa_{21} c_{2, t-1}+\cdots+\kappa_{2 L_{i}} c_{2, t-L_{t}},
\end{aligned}
$$

where $c_{1 t}$ and $c_{2 t}$ are the acquisitions of goods, the $\kappa_{i j}$ determine the extent to which past acquisitions of goods provide services (and hence utility) in the current period, and $L_{c}$ is the lag length. If $L_{c}=0$, then the utility function collapses to the standard time-separable case where $c_{1 t}^{*}=c_{1 t}$ and $c_{2 t}^{*}=c_{2 t}$. If the nonseparability parameters $\kappa_{i j}$ are positive, then past acquisitions of goods provide services today. If they are negative, then there is habit persistence as in Sundaresan (1989) and Constantinides (1990). Other patterns are possible as well. Recent acquisitions of goods can provide services today, while acquisitions further in the past contribute to habit persistence, as in Heaton (1993). 
The justification for using the form of preferences given above lies in the very strong evidence provided by Eichenbaum and Hansen (1990), Eichenbaum, Hansen, and Singleton (1988), Dunn and Singleton (1986), and Gallant and Tauchen (1989), that nonseparabilities must be introduced in order to fit asset returns and consumption series. More recently, Hansen and Jagannathan (1991) provide evidence from the U.S. stock market that habit persistence may be capable of explaining the risk premia that we observe in those markets.

\subsection{Introduction of money}

The manner in which money is introduced into the model is important. Lucas (1982) uses the cash-in-advance constraint, which allows money to be valued in equilibrium. Agents in Lucas's setting first enter the asset market with complete knowledge of the current state. If any security earning a positive nominal return exists, then agents will hold currency in exactly the amount needed to carry out their purchases of consumption goods; that is, the cash-in-advance constraint will always bind. This setup implies a unitary velocity of money.

Primarily to get around the implication of unitary velocity, Svensson (1985) changes the timing with which information is released to the agents. In his economy, agents decide on their cash balances before the current state is known. This change in timing gives rise to a nonunitary velocity, which coexists with positive interest rates.

To accommodate nonunitary velocity of money we introduce money as in Baumol (1952), Park (1985), Feenstra (1986), Marshall (1988), and Bansal (1990) via a transaction costs technology. The underlying justification for transactions costs is that acquisition of goods is costly both in terms of resources and time. Money, by its presence, economizes on these costs and hence is valued in equilibrium.

Transaction costs, $\psi(c, m)$, in our model depend on the amount of goods consumed, $c$, and the magnitude of real balances, $m$, held by the consumer in the trading period. The functional form we use for transaction costs technology is

$$
\psi(c, m)=\psi_{0} c^{\alpha} m^{1-\alpha},
$$

where $\psi_{0}>0$ and $\alpha>1$. The cash-in-advance constraint as given in Svensson (1985) is a special case of this transaction costs technology which satisfies $\psi(c, m)=$ $\infty$ for $c>m$ and $\psi(c, m)=0$ for $c \leqslant m$. The transaction costs technology above delivers a cash-in-advance constraint as $\alpha \rightarrow \infty$.

In the transaction cost model, we use a timing convention for selecting money balances similar to that of Svensson's (1985) cash-in-advance model to allow the return to holding money to be random with respect to current information. If the Lucas (1982) timing convention were followed, then the agent would determine his money holdings in the same period that they would be spent in the goods market, so the payoff to holding money would be completely certain. 


\subsection{The consumer's problem}

The consumer's problem is to maximize expected utility $\mathrm{E}_{0} \sum_{t=0}^{\infty} \beta^{t} U\left(c_{1 t}^{*}, c_{2 t}^{*}\right)$ by choosing $c_{1 t}, c_{2 t}, M_{1, t+1}, M_{2, t+1}, b_{1, t+1}^{k}$, and $b_{2, t+1}^{k}, k=1, \ldots, N_{a}$, at time $t$ subject to a sequence of budget constraints,

$$
\begin{aligned}
P_{1 t} & {\left[c_{1 t}+\psi\left(c_{1 t}, m_{1 t}\right)\right]+e_{t} P_{2 t}\left[c_{2 t}+\psi\left(c_{2 t}, m_{2 t}\right)\right] } \\
& +\sum_{k=1}^{N_{a}}\left(1 / R_{1 t}^{k}\right) b_{1, t+1}^{k}+\sum_{k=1}^{N_{a}}\left(f_{t}^{k} / R_{1 t}^{k}\right) b_{2, t+1}^{k}+M_{1, t+1}+e_{t} M_{2, t+1} \\
\leqslant & \sum_{k=1}^{N_{a}}\left(1 / R_{1 t}^{k-1}\right) b_{1 t}^{k}+\sum_{k=1}^{N_{a}}\left(f_{t}^{k-1} / R_{1 t}^{k-1}\right) b_{2 t}^{k}+M_{1 t}+e_{t} M_{2 t} \\
& +P_{1 t} w_{1 t}+e_{t} P_{2 t} w_{2 t}+q_{1 t}+e_{t} q_{2 t} .
\end{aligned}
$$

Here, $P_{1 t}$ and $P_{2 t}$ are current prices of consumption goods $c_{1 t}$ and $c_{2 t}$ in the units of the respective country's currency. $M_{1, t+1}$ and $M_{2, t+1}$ are the stocks of currency in the two countries carried forward from period $t$ to $t+1$. Real money balances, $m_{1 t}=M_{1 t} / P_{1 t}$ and $m_{2 t}=M_{2 t} / P_{1 t}$, are defined in terms of beginning of period money holdings, $b_{1, t+1}^{k}$ and $b_{2, t+1}^{k}$ are the agent's holdings of risk-free claims to the currencies of countries 1 and 2 in period $t+k$. Claims on country 1's currency are made by trading pure discount bonds with gross $k$-period interest rates, $R_{1 t}^{k}$. Claims on country 2's currency are made by trading forward contracts in the currency market, where $e_{t}$ is the spot exchange rate, and $f_{t}^{k}$ is the $k$-period forward exchange rate, with both rates defined in units of country 1's currency per unit of country 2 's currency; $w_{1 t}$ and $w_{2 t}$ are the stochastic endowments of goods within the two countries. Lump sum transfers of $q_{1 t}$ and $q_{2 t}$ units of currency are made by the government at time $t$. These transfers are known to the agent at the beginning of period $t$, but can be used for carrying out transactions only in period $t+1$.

The stationary decision problem facing the agent delivers the following Euler equations for the asset holdings $M_{1, t+1}$ and $M_{2, t+1}$ :

$$
\mathrm{E}_{t}\left[M U_{c_{i t}}-\beta M U_{c_{i, t+1}}\left(\frac{P_{i t}}{P_{i, t+1}}\right)\left(\frac{1+\psi_{c_{i t}}}{1+\psi_{c_{i, t+1}}}\right)\left(1-\psi_{m_{t, t+1}}\right)\right]=0, \quad i=1,2,
$$

and for $b_{1, t+1}^{k}$ and $b_{2, t+1}^{k}$.

$$
\begin{aligned}
& \mathrm{E}_{t}\left[M U_{c_{1 t}}-\beta^{k} M U_{c_{1, t+k}}\left(\frac{P_{1 t}}{P_{1, t+k}}\right)\left(\frac{1+\psi_{c_{1 t}}}{1+\psi_{c_{1, t+k}}}\right) R_{1 t}^{k}\right]=0, \\
& \mathrm{E}_{t}\left[M U_{c_{2 t}}-\beta^{k} M U_{c_{2, t+k}}\left(\frac{P_{2 t}}{P_{2, t+k}}\right)\left(\frac{1+\psi_{c_{2 t}}}{1+\psi_{c_{2, t+k}}}\right)\left(e_{t} R_{1 t}^{k} / f_{t}^{k}\right)\right]=0,
\end{aligned}
$$

where $M U_{c_{t \prime}}$ is the marginal utility of $c_{i t}$, and $\psi_{c_{t \prime}}$ and $\psi_{m_{t \prime}}$ are the derivatives of transaction costs, $\psi\left(c_{i t}, m_{i t}\right)$, with respect to the first and second arguments, 
respectively. Transactions costs modify the returns to the two monies, $M_{1 t}$ and $M_{2 t}$. We would expect $P_{1 t} / P_{1, t+1}$ to be the return at time $t+1$ for carrying forward an extra unit of country one's currency today. However, because of transaction costs, every extra unit of currency carried forward also lowers transaction costs in the next period by a real amount, $-\psi_{m_{t, t+1}}$, so the total return is given by $\left[\left(1-\psi_{m_{t, t+1}}\right) P_{i t} / P_{i, t+1}\right]$.

The model also delivers an intratemporal restriction on the choice of goods $c_{1 t}$ and $c_{2 t}$,

$$
e_{t}=\mathrm{E}_{t}\left[\left(\frac{M U_{c_{2 t}}}{M U_{c_{1 t}}}\right)\left(\frac{P_{1 t}}{P_{2 t}}\right)\left(\frac{1+\psi_{c_{1 t}}}{1+\psi_{c_{2 t}}}\right)\right] .
$$

In maximizing utility, the consumer faces an exogenous stochastic process that governs the evolution of money growth and endowment growth in the two countries. We define the operator $d$ to create the ratio of the value of a variable in one period to its value in the previous period, as, for example, $d M_{1 t}=M_{1 t} / M_{1, t-1}$. Using this operator, we specify a driving process for the exogenous state vector $S_{t}=$ $\left(d M_{1 t}, d M_{2 t}, d w_{1 t}, d w_{2 t}\right)$ of the form

$$
\log S_{t}=a_{0}+A \log S_{t-1}+u_{t},
$$

where $u_{t}$ is iid $\mathrm{N}(0, \Omega), a_{0}$ is a $4 \times 1$ vector, and $A$ and $\Omega$ are $4 \times 4$ matrices.

\subsection{Solution procedure}

The parameter vector of the structural economic model is

$$
\lambda=\left(\beta, \gamma, \delta, \psi_{0}, \alpha, \kappa_{11}, \ldots, \kappa_{1 L_{c}}, \kappa_{21}, \ldots, \kappa_{2 L_{c}}, a_{0}^{\prime}, \operatorname{vec}(A)^{\prime}, \operatorname{vech}\left(\Omega^{1 / 2}\right)^{\prime}\right)^{\prime} .
$$

Given a value of $\lambda$, we use the method of parameterized expectations to solve the model in the sense of determining simulated realizations of the variables that satisfy the Euler equations. Bansal, Gallant, Hussey, and Tauchen (1993) contains a complete description of how the method is applied to this problem, so we give only a brief description of the solution strategy here.

In solving the model, we first impose the market clearing conditions

$$
c_{i t}+\psi\left(c_{i t}, m_{i t}\right)=w_{i t}, \quad M_{i, t+1}=q_{i t}+M_{i t},
$$

$i=1,2$, on the Euler equations. Using the definition of the velocity of money, $V_{i t}=$ $c_{i t} P_{i t} / M_{i t}, i=1,2$, we note that the first two Euler equations can be expressed in terms of only money and endowment growths and velocities. The method of parameterized expectations is used to determine the two equilibrium velocity processes from these equations. Using these velocity processes, we can calculate directly consumption growth, $d c_{i t}$, and inflation, $d P_{i t}$, for the two countries, and we can use the method of parameterized expectations with the remaining Euler equations to determine the equilibrium $k$-period interest rates in country $1, R_{1 t}^{k}$, the premium of the $k$-period forward rate over the spot rate, $f_{t}^{k} / e_{t}$, and exchange rate growth, $d e_{t}$. 


\subsection{Cash-in-advance specification}

To gain some understanding of the contribution made by the manner in which money is introduced in our model, we also estimate a cash-in-advance version of the model that uses the timing convention for money adopted in Lucas (1982). The choice of that particular form of the cash-in-advance constraint is motivated by its simplicity and by the quantitative results of Hodrick, Kocherlakota, and Lucas (1991) who show that in practice the cash-in-advance constraint almost always binds. Because of the differences in timing conventions, the transaction cost model and the cash-in-advance model that we fit are nonnested.

The Lucas (1982) model is numerically solved by taking as the market clearing conditions

$$
c_{i t}=w_{i t}, \quad M_{i, t+1}=q_{i t}+M_{i t},
$$

and imposing a binding cash-in-advance constraint $p_{i t}=M_{i, t+1} / w_{i t}$. (In this model $M_{i, t+1}$ can now be used to purchase goods in period $t$; we maintain the dating of this variable as $t+1$, however, to keep the notation consistent between this model and the transaction cost model.) These market clearing restrictions allow us to write the Euler equations for the nominally risk-free bonds, $b_{i, t+1}^{k}, i=1,2$, and the growth rate of the exchange rate, $d e_{t}$, entirely in terms of conditional expectations of functions of past, current, and future realizations of the exogenous state variables. These Euler equations can be numerically solved by the method of parameterized expectations without any iterating. The simplification in the solution procedure is achieved because there is no wedge between $c_{i t}$ and $w_{i t}$, and thus no endogenous state variables, as there were in the transaction cost model.

\section{Nonparametric structural estimation}

\subsection{Linkage between the model and the data}

The structural model defines, for each value of $\lambda$, a nonlinear mapping from the strictly exogenous process $\left\{S_{t}\right\}$ to an output process $\left\{U_{t}\right\}$. The exogenous process is $S_{t}=\left(d M_{1 t}, d M_{2 t}, d w_{1 t}, d w_{2 t}\right)^{\prime}$, a $4 \times 1$ vector containing the gross money growth rates and gross endowment growth rates for each country. The output process is

$$
U_{t}=\left(d M_{1 t}, d M_{2 t}, d w_{1 t}, d w_{2 t}, d c_{1 t}, d c_{2 t}, d P_{1 t}, d P_{2 t}, R_{1 t}^{4}, f_{t}^{4} / e_{t}, d e_{t}\right)^{\prime},
$$

which is an $11 \times 1$ vector containing the elements of $S_{t}$ along with the gross consumption growth rates, the gross inflation rates, the four-period interest rate in country 1 , the ratio of the four-period forward exchange rate to the spot rate, and the gross growth rate of the spot exchange rate. It proves convenient to include the elements of $S_{t}$ in the output process, thereby taking the mapping from $S_{t}$ to those elements as the identity map. The particular set of variables comprising the remaining elements 
of $U_{t}$ are those endogenous variables that turn out to be of interest for various aspects of the analysis of the model and the empirical work. Relative to the model, this set is not minimal, as there are redundancies; relative to the observed data, the set contains more variables than are actually observed at a weekly frequency.

The mapping from $\left(\left\{S_{t}\right\}, \lambda\right)$ to the endogenous elements of $U_{t}$ is defined by the solution to the nonlinear rational expectations model. As noted, we use Marcet's method of parameterized expectations (Marcet, 1991; den Haan and Marcet, 1990) to approximate the map. This method has some convenient properties for our particular setup, since we only need simulated realizations from the model and not the full set of decision rules. In what follows $\left\{U_{t}^{i}\right\}$ denotes a realization of the output process given $\lambda$ and a realization of $\left\{S_{t}\right\}$.

Not all elements of $U_{t}$ are available on a weekly frequency, so our empirical strategy is to use latent variable and simulation methods to estimate the model. In particular, weekly endowment growth rates $\left(d w_{1 t}, d w_{2 t}\right)$ are not tabulated. Although weekly money supply data are available for the U.S., these data are highly volatile and contaminated by measurement error and high-frequency seasonal noise, and appear to be of limited usefulness. Our strategy is to treat the elements of $S_{t}$ as unobserved processes generated by a four-dimensional linear VAR(1) model with Gaussian errors. Likewise, consumption and price level data are not recorded weekly, so we do not use $d c_{i t}$ and $d P_{i t}$ in the empirical work.

Widely available on a weekly basis are high-quality observations on financial market prices, i.e., payoff data, and so we concentrate on these series in the estimation. We utilize weekly observations on three raw series: $S P O T_{t}$, the spot exchange rate (in \$ per DM), FORWARD $D_{t}^{4}$, the 30-day forward rate (in \$ per DM), TBILL $L_{t}^{4}$, the one-month treasury bill interest rate, computed from the term structure, and quoted on a bank discount basis. More details are available about the raw data in Section 4 below. From the raw series we form a $3 \times 1$ process $y_{t}=\left(y_{1 t}, y_{2 t}, y_{3 t}\right)^{\prime}$ with

$$
\begin{aligned}
& y_{1 t}=100 * \log \left(\text { SPOT }_{t} / \text { SPOT }_{t-1}\right), \\
& y_{2 t}=100 * \log \left(\text { FORWARD }_{t}^{4} / \text { SPOT }_{t}\right), \\
& y_{3 t}=\text { TBILL }_{t}^{4} .
\end{aligned}
$$

Exploratory empirical work, described in Section 4, indicates that $\left\{y_{t}\right\}$ is reasonably taken as a strictly stationary process, while the levels of the exchange rate series are nonstationary.

The correspondence between the elements of $y_{t}$ and those of the output vector $U_{t}$ are as follows. Country 1 is the U.S. and country 2 is Germany. Given a simulated realization $\left\{U_{t}^{\lambda}\right\}$ from the model, the corresponding $\left\{y_{t}^{\lambda}\right\}$ is computed as

$$
\begin{aligned}
& y_{1 t}^{\lambda}=100 * \log \left(d e_{t}^{\lambda}\right), \\
& y_{2 t}^{\lambda}=100 * \log \left(f_{t}^{4} / e_{t}\right), \\
& y_{3 t}^{\lambda}=100 *(360 / 30)\left[1-\left(1 / R_{1 t}^{4 \lambda}\right)\right] .
\end{aligned}
$$


The expression for $y_{3 t}^{\lambda}$ converts $1 / R_{1 t}^{4 \lambda}$, which is the price at time $t$ of $\$ 1$ in period $t+4$, to an annualized interest rate using the bank discount formula customarily applied to treasury bill prices (Stigum, 1990, p. 66).

\subsection{The estimation method}

The observed process is $\left\{y_{t}\right\}$ and the simulated process is $\left\{y_{t}^{\lambda}\right\}$ as defined above. The $\left\{y_{t}\right\}$ process is computed directly from the raw data while $\left\{y_{t}^{\hat{\lambda}}\right\}$ is computed using the structural model of Section 2. We assume the model to be 'true', in the sense that there is a particular value, say $\lambda_{0}$, of the structural parameter vector and a realization, say $\left\{S_{0 t}\right\}$, of the exogenous vector such that the observed $\left\{y_{t}\right\}$ is obtained from $\left(\left\{S_{0 t}\right\}, \lambda_{0}\right)$ in exactly the same manner that the model generates $\left\{y_{t}^{\prime}\right\}$ from $\left(\left\{S_{t}\right\}, \lambda\right)$.

The setup is thus one in which it is possible to use simulation methods to compute predicted probabilities and expectations under the model, but at the same time it is computationally intractable to compute the likelihood function of a sample as a function of the parameters of the model. In broad terms, the setup is analogous to the situation described in Duffie and Singleton (1993), Ingram and Lee (1991), and elsewhere. Common practice is to use a simulated method of moments estimator of $\lambda_{0}$ based upon certain a priori selected moments of the data.

The estimation strategy of this paper is different and starts from the point of view that the structural model should be forced to confront all empirically relevant aspects of the observed process. More to the point, the observed process $\left\{y_{t}\right\}$ is strictly stationary and possibly nonlinear. Its dynamics are completely described by the onestep-ahead conditional density $f\left(y_{t} \mid\left\{y_{t-j}\right\}_{j=1}^{\infty}\right)$. The conditional density $f(\cdot \mid \cdot)$ is not an element of any finite-dimensional parametric family, so no finite-dimensional vector of fixed length can characterize the process. Nonetheless, it is still possible to estimate $f(\cdot \cdot)$ consistently using nonparametric methods. Let $\hat{f}(\cdot \cdot \cdot)$ denote a nonparametric estimate computed from a realization $\left\{y_{t}\right\}_{t=t_{0}}^{n}$. The estimator $\hat{f}(\cdot \mid \cdot)$ defines what is empirically relevant about the process and thereby provides a comprehensive standard of reference upon which to match the economic model to the data.

One way to implement this notion of matching would be to define the estimator $\hat{\lambda}$ to be the value of $\lambda$ that minimizes the distance (under a suitable metric) between the nonparametric estimator fitted to the observed data and fitted to the simulated data. Specifically, if $\hat{f}(\cdot \mid \cdot)$ denotes the estimator applied to $\left\{y_{t}\right\}_{t=t_{0}}^{n}$ and $\tilde{f}_{i}(\cdot \mid \cdot)$ denotes the same estimator applied to a simulated realization $\left\{y_{\tau}^{\dot{\alpha}}\right\}_{\tau=\tau_{0}}^{\pi_{0}}$, then $\hat{\lambda}=\arg \min _{\lambda}\left\|\hat{f}(\cdot \cdot)-\tilde{f}_{\lambda}(\cdot \cdot \cdot)\right\|$. Here $\|\cdot\|$ is a suitable norm on densities, e.g., a Sobolev norm. This method would be direct analogue of Manski's (1983) estimator for independent data. It is computationally intractable, though, because of the infeasibility of re-computing $\tilde{f}_{\lambda}(\cdot \cdot \cdot)$ over a wide range of $\lambda$ 's, as would be required to do the minimization. With some modification, however, this concept can be implemented in a computationally tractable manner. 
The key idea is to use the scoring function of the SNP estimator of Gallant and Tauchen $(1989,1992 a)$ which provides a consistent nonparametric estimator of the conditional density under mild regularity conditions. This use of the nonparametric fit to define the criterion of estimation motivates our choice of the term 'nonparametric structural estimator'. The Gallant-Tauchen estimator is a truncation estimator based on a series expansion that defines an hierarchy of increasingly complex models. The estimator $\hat{f}(\cdot \cdot)=f_{K_{n}}\left(\cdot \mid \cdot \hat{\theta}_{K_{n}}\right)$ is characterized by an auxiliary parameter vector $\hat{\theta}_{K_{n}}$ that contains the coefficients of the expansion; the subscript $K_{n}$ denotes the $K_{n}$ th model in the hierarchy. (See Section 4.2 below.) The length of $\hat{\theta}_{K_{n}}$ depends on the model. In practice, $K_{n}$ is determined by a model selection criterion that slowly expands the model with sample size $n$ and thereby ensures consistency. For the $K_{n}$ th model in the hierarchy, the corresponding $\hat{\theta}_{K_{n}}$ is given by

$$
\hat{\theta}_{K_{n}}=\arg \max _{\theta_{K} \in \Theta_{K}}\left\{\frac{1}{n} \sum_{t=1}^{n} \log \left[f\left(y_{t} \mid x_{t-1}, \theta_{K}\right]\right\},\right.
$$

and thus $\hat{\theta}_{K_{n}}$ solves the first-order condition

$$
\frac{\partial}{\partial \theta_{K_{n}}} \mathscr{L}_{K_{n}}\left(\left\{y_{t}\right\}_{t=t_{0}}^{n}, \hat{\theta}_{K_{n}}\right)=0,
$$

where $\mathscr{L}_{K_{n}}(\cdot)$ is sample log-likelihood (for model $K_{n}$ ).

The nonparametric structural estimator is defined by mimicking the first-order condition in the simulation. Subject to identifiability and regularity conditions, a consistent estimator is available by choosing $\hat{\lambda}$ so as to make the same condition hold (as closely as possible) in the simulation

$$
\frac{\partial}{\partial \theta_{K_{n}}} \mathscr{L}_{K_{n}}\left(\left\{y_{\tau}^{\lambda}\right\}_{\tau=\tau_{0}}^{\mathscr{T}}, \hat{\theta}_{K_{n}}\right) \approx 0 .
$$

The left-hand side is the gradient of the log-likelihood function evaluated at a simulated realization, $\left\{y_{\tau}^{\lambda}\right\}_{\tau=\tau_{0}}^{\mathbb{T}}$, and at the $\hat{\theta}_{K_{n}}$ determined by fitting the $K_{n}$ th SNP model to the observed data $\left\{y_{t}\right\}_{t=t_{0}}^{n}$. When the length of $\lambda$, denoted by $\ell_{\lambda}$, is less than the length of $\theta_{K}$, denoted $\ell_{K}$, then the model is overidentified (under the order condition) and a GMM criterion is used to minimize the length of the left-hand side with respect to a suitable weighting matrix.

The nonparametric structural estimator $\hat{\lambda}$ is the solution of the GMM estimation problem

$$
\hat{\lambda}=\arg \min _{\lambda \in \Lambda}\left[\tilde{s}_{n}(\lambda)\right],
$$

where

$$
\tilde{s}_{n}(\lambda)=n \tilde{g}\left(\lambda, \hat{\theta}_{K_{n}}\right)^{\prime} W_{n} \tilde{g}\left(\lambda, \hat{\theta}_{K_{n}}\right),
$$


and where

$$
\begin{aligned}
& \tilde{g}\left(\lambda, \hat{\theta}_{K n}\right)=\frac{\partial}{\partial \theta_{K_{n}}} \mathscr{L}_{K_{n}}\left(\left\{y_{\tau}^{\lambda}\right\}_{\tau=\tau_{0}}^{\mathbb{Z}}, \hat{\theta}_{K_{n}}\right), \\
& W_{n}=\left\{\frac{1}{n} \sum_{t=1}^{n}(\partial / \partial \theta) \log \left[f\left(y_{t} \mid x_{t-1}, \hat{\theta}_{K_{n}}\right)\right]\left(\partial / \partial \theta^{\prime}\right) \log \left[f\left(y_{t} \mid x_{t-1}, \hat{\theta}_{K_{n}}\right]\right)\right\}^{-1} .
\end{aligned}
$$

The matrix $W_{n}$ is the natural estimate of the inverse of the information matrix based on the gradient-outer-product formula.

As long as $K_{n}=K$ is left fixed as $n \rightarrow \infty$, then the asymptotic theory of $\hat{\lambda}$ can be derived by combining the asymptotic theory of misspecified finite-dimensional parametric models with the theory of GMM estimation of Hansen (1982). Gallant and Tauchen (1992b) develop this theory - which is entirely parametric - under a variety of estimation environments for a class of estimators that subsumes $\hat{\lambda}$. In their terminology, the SNP model is the 'score generator.' A good score generator provides a close statistical approximation to the conditional distribution of the data. Their treatment includes the stationary setup of this paper and more complicated setups with nonstationarity and unconditional heterogeneity. It presumes that $\mathscr{T}$ is large enough that the Monte Carlo integral approximates the analytical integral to within a negligible error of the same sort as is made in computing any mathematical expression on a digital computer. Their results state that $\hat{\lambda}$ is consistent, asymptotically normal, and $\tilde{s}_{n}(\hat{\lambda})$ is asymptotically $\chi^{2}\left(\ell_{K}-\ell_{\dot{\lambda}}\right)$. The asymptotic variance of $\hat{\lambda}$ depends upon the expectation under the model of the gradient with respect to $\lambda$ of the expected SNP score. This gradient is difficult to approximate numerically and standard errors thereby hard to compute, so in practice statistical inference works off the limiting distribution of the minimized objective function.

Interestingly, the approach just described defines a consistent and asymptotically normal estimator irrespective of the particular SNP model used, so long as $\ell_{K} \geqslant \ell_{i}$ and an identification condition is met. For instance, as can easily be checked, if one uses the lowest-order SNP model, which is a VAR with Gaussian errors, then this estimator is essentially an Ingram-Lee (1991) estimator using first and second moments to define the criterion function. Using the next model up in the SNP hierarchy, which is a Gaussian ARCH model, introduces additional moments based on the serial covariance structure of the squared residuals. Using successively larger SNP models increases the length of $\theta_{K}$ and thereby introduces additional scoring indicators to capture skewness, kurtosis, and additional conditional heterogeneity beyond the ARCH.

In practice, we implement the estimator using the SNP model that emerges from the specification search in the nonparametric estimation of $f(\cdot \mid \cdot)$. This selection rule forces the scoring function to be appropriate for the particular sample at hand. The score function of the fitted SNP model contains just those indicators important to fit the data, and no more. The choice of $K$ is thus data-determined, which, together with the consistency properties of SNP estimations, gives the estimator 
its nonparametric properties. When $K$ grows with sample size, either adaptively or deterministically, and $\theta^{o}$ is regarded as the truncation of an infinite-dimensional parameter, inferences using standard asymptotics are asymptotically valid and have a nonparametric interpretation in the situations considered by Andrews (1991), Eastwood (1991), Gallant and Souza (1991). However, the processes and estimators they studied are simpler than those discussed here, and their results have not been extended to our more complicated setting.

\section{Data and SNP estimation}

\subsection{Basic data}

The raw data are weekly (Friday) observations on three series:

$$
\begin{aligned}
& \text { SPOT }_{t}=\text { spot exchange rate, } \$ / \mathrm{DM}, \\
& \text { FORWARD }_{t}^{4}=30 \text {-day forward rate, } \$ / \mathrm{DM}, \\
& \operatorname{TBILL}_{t}^{4}=\text { one-month treasury bill rate. }
\end{aligned}
$$

The sample period is Friday, January 3, 1975, to Friday, December 28, 1990, which yields 835 raw observations. If a Friday is a holiday, then data for the nearest preceding nonholiday is used. To ensure appropriate alignment, daily observations on each series were collected and checked for accuracy, and then the weekly data series extracted.

For the years 1978-90, the spot and forward exchange rate data are New York opening quotations, kindly supplied by Bankers Trust, New York, NY. For the years 1975-77, the exchange rate data are mid-day Chicago quotations taken from issues of the IMM Yearbook.

The one-month treasury bill rate was obtained from the Federal Reserve Board. This rate, which is calculated on a daily basis, is computed from the average price in the secondary market for the treasury bill that matures four weeks from the next Thursday. The price is the average from five dealers of the bid prices at approximately 4 p.m. Eastern time. Because there is a two-business-day settlement period in the secondary market, this rate is actually a forward rate on an investment whose term is between 27 days and 31 days, depending upon the day of the week. In particular, for our Friday series, the buyer of the bill on Friday would normally take delivery the subsequent Tuesday. Hence our Friday one-month treasury bill rate is most precisely characterized as a four-day forward rate for a 30-day investment.

Extensive preliminary work using Dickey-Fuller unit root analysis (Dickey and Fuller, 1979) indicated that both $\log \left(S_{P O T}\right)$ and $\log \left(F O R W A R D_{t}^{4}\right)$ are integrated, i.e., $I(1)$ processes, while their first differences are $I(0)$. The analysis also strongly suggested that $\log \left(F O R W A R D_{t}^{4} / S P O T_{t}\right)$ is $I(0)$, reflecting cointegration between the spot and forward rates in the form of a stationary forward/spot premium. The 
Table 1

Summary statistics

\begin{tabular}{lllll}
\hline & Mean & Std. dev. & Skewness & Kurtosis \\
\hline$y_{1}$ & 0.055 & 1.496 & 1.126 & 6.172 \\
$y_{2}$ & 0.291 & 0.191 & 0.006 & 4.672 \\
$y_{3}$ & 7.730 & 2.696 & 23.565 & 4.077 \\
\hline
\end{tabular}

Autocorrelation function, $\operatorname{corr}\left(y_{i, t}, y_{j, t-k}\right)$

\begin{tabular}{rrrrrrr} 
& \multicolumn{1}{c}{$i, j$} & & & & & \\
\cline { 6 - 7 } & 1,1 & 1,2 & 2,2 & 1,3 & 2,3 & 3,3 \\
\hline-5 & -0.061 & -0.120 & 0.789 & -0.140 & 0.641 & 0.924 \\
-4 & 0.045 & -0.118 & 0.840 & -0.142 & 0.661 & 0.943 \\
-3 & 0.016 & -0.127 & 0.886 & -0.137 & 0.676 & 0.956 \\
-2 & 0.034 & -0.127 & 0.927 & -0.138 & 0.689 & 0.970 \\
-1 & 0.021 & -0.145 & 0.965 & -0.136 & 0.698 & 0.985 \\
0 & 1.000 & -0.128 & 1.000 & -0.126 & 0.702 & 1.000 \\
1 & 0.021 & -0.105 & 0.965 & -0.096 & 0.690 & 0.985 \\
2 & 0.034 & -0.107 & 0.927 & -0.077 & 0.670 & 0.970 \\
3 & 0.016 & -0.094 & 0.886 & -0.082 & 0.645 & 0.956 \\
4 & 0.045 & -0.056 & 0.840 & -0.070 & 0.621 & 0.943 \\
5 & -0.061 & -0.050 & 0.789 & -0.063 & 0.595 & 0.924 \\
\hline
\end{tabular}

$$
y_{1 t}=100 * \log \left(\operatorname{SPOT}_{t} / \text { SPOT }_{t-1}\right), y_{2 t}=100 * \log \left(\text { FORWARD }_{t}^{4} / \text { SPOT }_{t}\right), y_{3 t}=\text { TBILL }_{t}^{4} .
$$

evidence was less clear for $T B I L L_{t}^{4}$, as test statistics typically lay between the 5 percent and 10 percent critical regions when $I(1)$ was taken as the null and $I(0)$ the alternative. In what follows we take $T B I L L_{t}^{4}$ as a stationary $I(0)$ process, which reflects both our strong a priori opinion that nominal interest rates are stationary and the low power characteristics of unit root tests when $I(1)$ is the null.

On the basis of this work, we elected to use the following three series as the basic series time series for model estimation and evaluation:

$$
\begin{aligned}
& y_{1 t}=100 * \log \left(\text { SPOT }_{t} / \text { SPOT }_{t-1}\right), \\
& y_{2 t}=100 * \log \left(\text { FORWARD }_{t}^{4} / \text { SPOT }_{t}\right), \\
& y_{3 t}=\text { TBILL }_{t}^{4} .
\end{aligned}
$$

Thus $y_{t}=\left(y_{1 t}, y_{2 t}, y_{3 t}\right)^{\prime}$ consists of logarithmic exchange rate growth, the log of the forward/spot premium, and the nominal interest rate. Since time is measured in units of weeks, both the forward rate and the interest rate are four-period financial instruments. There are 834 observations on $y_{t}$.

Table 1 shows some basic summary statistics on the three series. The exchange rate is seen to be highly volatile, with the standard deviation of weekly movements being almost 1.50 percent per week. The distribution of weekly exchange rate movements is slightly right-skewed and leptokurtic, i.e., its kurtosis exceeds 3.0 , the value for 


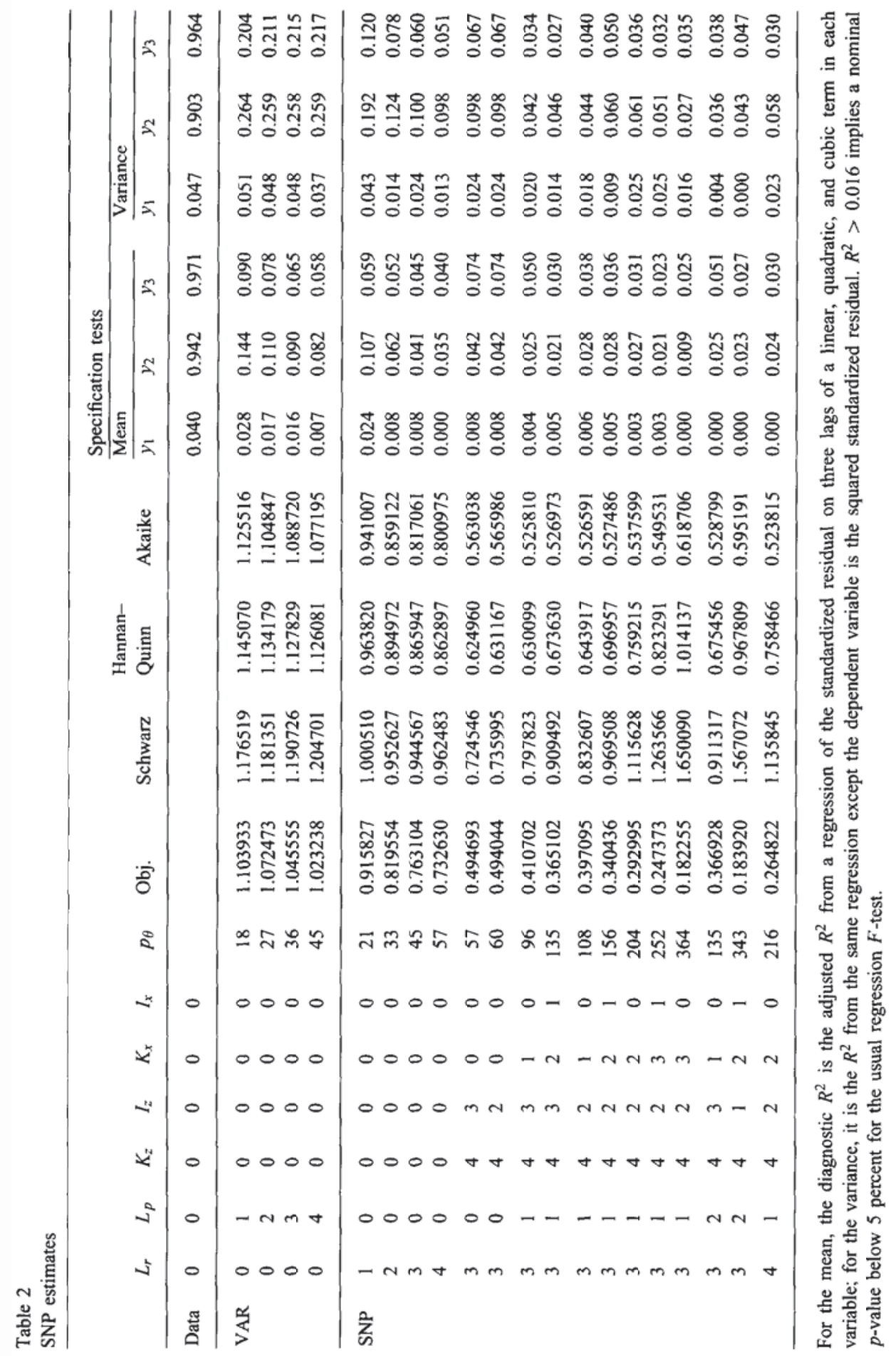


Table 3

Tests for unbiasedness of the forward rate

\begin{tabular}{lrcc}
\hline$J$ & d.f. & Chi-square & $p$-value \\
\hline $\mathscr{F}_{J t}^{1}$ & 2 & & \\
0 & 4 & 2.4422 & 0.2949 \\
3 & 6 & 5.4334 & 0.2456 \\
5 & & 8.7993 & 0.1852 \\
$\mathscr{F}_{J t}^{2}$ & 3 & & \\
0 & 7 & 11.4488 & 0.0095 \\
3 & 11 & 18.7981 & 0.0088 \\
5 & & 21.6918 & 0.0269 \\
$\mathscr{F}_{J t}^{3}$ & 4 & & \\
0 & 10 & 11.4628 & 0.0218 \\
3 & 20.7542 & 0.0229 \\
\hline
\end{tabular}

The orthogonality condition is

$$
\mathrm{E}\left(\left[\log \left\{\text { SPOT }_{t+4}\right\}-\log \left\{\text { FORWARD }_{t}^{4}\right\}\right] z_{t} \mid \mathscr{F}_{J_{t}}^{i}\right)=0 .
$$

where

$$
\begin{aligned}
& z_{t} \in \mathscr{F}_{J t}^{i}, \\
& \mathscr{F}_{J t}^{1}=\left\{1, \log \left(\text { SPOT }_{t-j}\right)-\log \left(\text { FORWARD }_{t-4-j}\right)\right\}_{j=0}^{J}, \\
& \mathscr{F}_{J_{t}}^{2}=\left\{1, \log \left(\text { SPOT }_{t-j}\right)-\log \left(\text { FORWARD }_{t-4-j}\right)\right. \\
&\left.\log \left(\text { SPOT }_{t-j}\right)-\log \left(\text { FORWARD }_{t-j}\right)\right\}_{j=0}^{J}, \\
& \mathscr{F}_{J t}^{3}=\left\{1, \log \left(\text { SPOT }_{t-j}\right)-\log \left(\text { FORWARD }_{t-4-j}\right)\right. \\
& \log \left(\text { SPOT }_{t-j}\right)-\log \left(\text { FORWARD }_{t-j}\right) \\
&\left.\log \left(\text { SPOT }_{t-j}\right)-\log \left(\text { SPOT }_{t-j-1}\right)\right\}_{j=0}^{J} .
\end{aligned}
$$

Reported variance estimates are computed using Parzen's weights with a lag length of 6 . Lag lengths of 3 and 9 were also tried with little difference in the results.

the normal distribution. The distribution of the log of the forward/spot ratio is symmetric and leptokurtic, while that of the one-month treasury bill rate is rightskewed and also leptokurtic. The autocorrelation function indicates that exchange rate movements are essentially white noise, while both the log of the forward/spot ratio and the one-month treasury bill rate are highly persistent with strong crossautocorrelation.

Table 3 provides a brief summary of tests for the unbiasedness of the forward rate as a predictor of the subsequent spot rate. Under certain restrictive notions of market efficiency, the forward rate can be argued to be an unbiased predictor of the spot rate. For the reasons discussed in Hansen and Hodrick (1983), the logarithmic form of the test is used here to free the results from the units of measurement. The test statistics pertain to the condition $\mathrm{E}\left[\log \left(S_{P O T_{t+4}}\right)-\log \left(F O R W A R D_{t}^{4}\right) \mid \mathscr{F}_{t}\right]=0$, for various information sets $\mathscr{F}_{t}$. The statistics are computed using a robust weighted 
variance estimate with Parzen's weights (Gallant, 1987, p. 446) and lag window lengths of 3,6 , and 9 ; we only report the results for a lag of 6 as there is little difference across the sets of results. [The forecast errors have an induced $M A(3)$ structure due to the overlapping intervals.

The reported tests fail to reject unbiasedness if the information set is restricted to lagged forecast errors; however, the tests sharply reject unbiasedness once the forward/spot premium is included in the model, which is in general consistent with the literature. These conclusions are clearly quite robust with respect to the lag window length. Rejection of unbiasedness is not surprising in view of theoretical models discussed in Section 2. These models provide an explicit theory that connects the risk premium to underlying deep parameters. Only under very special circumstances - risk-neutral agents and zero inflation - will unbiasedness emerge as the prediction from equilibrium models.

We now proceed to SNP estimation of the conditional density of $y_{t}$ given past $y$ 's. Discussion of the estimation is preceded by an overview of the SNP method.

\subsection{Seminonparametric ( SNP) estimators}

The SNP method is based on the notion that a Hermite expansion can be used as a general purpose nonparametric estimator of a density function. Letting $z$ denote an $M$-vector, the particular Hermite expansion employed has the form $f(z) \propto$ $[P(z)]^{2} \phi(z)$ where $P(z)$ denotes a multivariate polynomial of degree $K_{z}$ and $\phi(z)$ denotes the density function of the (multivariate) Gaussian distribution with mean zero and the identity matrix as its variance-covariance matrix. The constant of proportionality is the divisor $\int[P(z)]^{2} \phi(z) \mathrm{d} z$ which makes $f(z)$ integrate to one. Because of this division, the density is a homogeneous function of the coefficients of the polynomial $P(z)$, and these coefficients can only be determined to within a scalar multiple. To achieve a unique representation, the constant term of the polynomial part is put to one.

The location/scale shift $y=R z+\mu$, where $R$ is an upper triangular matrix and $\mu$ is an $M$-vector, followed by a change of variables leads to a parameterization that is easy to interpret: $f(y \mid \theta) \propto\left\{P\left[R^{-1}(y-\mu)\right]\right\}^{2}\left\{\phi\left[R^{-1}(y-\mu)\right] /|\operatorname{det}(R)|\right\}$. Because $\left\{\phi\left[R^{-1}(y-\mu)\right] /|\operatorname{det}(R)|\right\}$ is the density function of the $M$-dimensional multivariate Gaussian distribution with mean $\mu$ and variance-covariance matrix $\Sigma=$ $R R^{\prime}$, and because the leading term of the polynomial part equals unity, the leading term of the entire expansion is the multivariate Gaussian density function; denote it by $n_{M}(y \mid \mu, \Sigma)$. When $K_{z}$ is put to zero, one gets $n_{M}(y \mid \mu, \Sigma)$ exactly. When $K_{z}$ is positive, one gets a Gaussian density whose shape is modified due to multiplication by a polynomial in the normalized error $z=R^{-1}(y-\mu)$. The shape modifications thus achieved are rich enough to accurately approximate densities from a large class that includes densities with fat $t$-like tails, densities with tails that are thinner than Gaussian, and skewed densities.

The parameters $\theta$ of $f(y \mid \theta)$ are made up of the coefficients of the polynomial 
$P(z)$ plus $\mu$ and $R$ and are estimated by maximum likelihood. Equivalent to maximum likelihood but more stable numerically is to estimate $\theta$ in a sample of size $n$ by minimizing $s_{n}(\theta)=(-1 / n) \sum_{t=1}^{n} \log \left[f\left(y_{t} \mid \theta\right)\right]$. If the number of parameters $p_{\theta}$ grows with the sample size $n$, then the true density and various features of it such as derivatives and moments are estimated consistently. Because the method is parametric yet has nonparametric properties, it is termed seminonparametric to suggest that it lies halfway between parametric and nonparametric procedures.

This basic approach is adapted to the estimation of the conditional density of a multiple time series that has a Markovian structure as follows. By a Markovian structure, one means that the conditional density of the $M$-vector $y_{t}$ given the entire past $y_{t-1}, y_{t-2}, \ldots$ depends only on $L$ lags from the past. For notational convenience, we collect these lags together in a single vector denoted as $x_{t-1}$ which has length $M \cdot L$. As above, a density is obtained by a location/scale shift $y_{t}=R z_{t}+\mu_{x}$ off a sequence of normalized errors $\left\{z_{t}\right\}$. Here $\mu_{x}$ is a linear function of $x_{t-1}$, specifically $\mu_{x}=b_{0}+B x_{t-1}$ where $b_{0}$ is $M \times 1$ and $\mathrm{B}$ is $M \times M \cdot L$, making the leading term of the expansion $n_{M}\left(y \mid \mu_{x}, \Sigma\right)$, which is a Gaussian vector autoregression or Gaussian VAR. In time series analysis, the $z_{t}$ are usually referred to as linear innovations. In order to permit the innovations to be conditionally heterogeneous, the coefficients of the polynomial $P(z)$ are, themselves, polynomials of degree $K_{x}$ in $x_{t-1}$. This polynomial is denoted as $P(z, x)$. When $K_{x}=0$, the $\left\{z_{t}\right\}$ are homogeneous, as the conditional density of $z_{t}$ does not depend upon $x_{t-1}$. When $K_{x}>0$, the $\left\{z_{t}\right\}$ are conditionally heterogeneous. The tuning parameter $K_{z}$ controls the extent to which the model deviates from normality while $K_{x}$ controls the extent to which these deviations vary with the history of the process.

To keep $K_{x}$ small when the data exhibit marked conditional heteroskedasticity, the leading term of the expansion can be put to a Gaussian ARCH rather than a Gaussian VAR. This is done by letting $R$ be a linear function of the absolute values of (the elements of) $L_{r}$ of the lagged $y_{t}$, centered and scaled to have mean zero and identity covariance matrix. This differs from the classical ARCH (Engle, 1982) which has $\Sigma_{x}$ depending on a linear function of squared lagged residuals; the SNP version of ARCH is more akin to the suggestions of Nelson (1991) and Davidian and Carroll (1987). The SNP specification is $\Sigma_{x}=R_{x} R_{x}^{\prime}$, with $\operatorname{vech}\left(R_{x}\right)=P_{0}+P_{1} \operatorname{abs}\left(x_{t-1}^{*}\right)$, where $P_{0}$ and $P_{1}$ are coefficient matrices of dimension $M \cdot(M+1) / 2 \times 1$ and $[(M \cdot(M+1) / 2)] \times M \cdot L$, respectively, $x_{t-1}^{*}$ is centered and rescaled $x_{t-1}$, and abs $\left(x_{t-1}^{*}\right)$ is the element-wise absolute value of $x_{t-1}^{*}$. The form of the conditional density becomes $f(y \mid x, \theta) \propto[P(z, x)]^{2} n_{M}\left(y \mid \mu_{x}, \Sigma_{x}\right)$ where $z=R_{x}^{-1}\left(y-\mu_{x}\right)$ and $\theta$ denotes the coefficients of the polynomial $P(z, x)$ and the Gaussian ARCH $n_{M}\left(y \mid \mu_{x}, \Sigma_{x}\right)$ collected together. The parameters are estimated by minimizing $s_{n}(\theta)=(-1 / n) \sum_{t=1}^{n} \log \left[f\left(y_{t} \mid x_{t-1}, \theta\right)\right]$.

We distinguish between the total number of lags under consideration, which is $L$, the number of lags in the $x$ part of the polynomial $P(z, x)$, which we denote by $L_{p}$, and the number of lags in $\Sigma_{x}$, which is $L_{r}$. The vector $x$ has length $M \cdot L$ where $L=\max \left(L_{r}, L_{p}\right)$. 
Table 4

Restrictions on the process $\left\{y_{t}\right\}$

\begin{tabular}{ll}
\hline Parameter setting & Characterization of $\left\{y_{t}\right\}$ \\
\hline$L_{r}=0, L_{p}=0, K_{z}=0, K_{x}=0$ & iid Gaussian \\
$L_{r}=0, L_{p}>0, K_{z}=0, K_{x}=0$ & Gaussian VAR \\
$L_{r}=0, L_{p}>0, K_{z}>0, K_{x}=0$ & non-Gaussian VAR, \\
& homogeneous innovations \\
$L_{r}>0, L_{p}=0, K_{z}=0, K_{x}=0$ & Gaussian ARCH \\
$L_{r}>0, L_{p}>0, K_{z}>0, K_{x}=0$ & non-Gaussian ARCH, \\
& homogeneous innovations \\
\hline
\end{tabular}

Large values of $M$ can generate a large number of interactions (cross-product terms) for even modest settings of degree $K_{z}$; similarly, for $M \cdot L_{p}$ and $K_{x}$. Accordingly, there are two additional tuning parameters, $I_{z}$ and $I_{x}$, to represent filtering out of these high-order interactions. $I_{z}=0$ means no interactions are suppressed, $I_{z}=1$ means the highest-order interactions are suppressed, namely those of degree exceeding $K_{z}-1$. In general, a positive $I_{z}$ means all interactions of order exceeding $K_{z}-I_{z}$ are suppressed; similarly for $K_{x}-I_{x}$.

To illustrate, consider a special case where $M=2, L_{r}=3, L_{p}=1, K_{z}=4$, $I_{z}=2, K_{x}=2$, and $I_{x}=1$. Then the polynomial $P(z, x)$ would take the form

$$
P(z, x)=\sum_{\lambda_{1}, \lambda_{2}} a\left(\lambda_{1}, \lambda_{2}, x\right) z_{1}^{\lambda_{1}} z_{2}^{\lambda_{2}}
$$

where the $a\left(\lambda_{1}, \lambda_{2}, x\right)$ are the coefficients of the polynomial in $z \in \Re^{2}$, and the sum is over all pairs of nonnegative integers $\left(\lambda_{1}, \lambda_{2}\right)$ such that $\lambda_{1}+\lambda_{2} \leqslant 4$, excluding the quartic interactions, $(1,3),(2,2)$, and $(3,1)$, and the cubic interactions, $(2,1)$ and $(1,2)$, suppressed by $I_{z}=2$. With $L_{r}=3$ and $L_{p}=1$, then the ARCH part would depend on $y_{t}$ back to lag 3 and the polynomial coefficient $a\left(\lambda_{1}, \lambda_{2}, x\right)$ back to lag 1. With $K_{x}=2$ and $I_{x}=1$, then the $a\left(\lambda_{1}, \lambda_{2}, x\right)$ would be quadratic functions of the elements of $x$, with all cross-products suppressed, since these are quadratic interactions.

In general, $L_{r}$ and $L_{p}$ determine the location/scale shift $y=R_{x} z_{t}+\mu_{x}$ and hence determine the nature of the leading term of the expansion. The number of lags in the location shift $\mu_{x}$ is the overall lag length $L$ which is the maximum of $L_{r}$ and $L_{p}$. The number of lags in the scale shift $R_{x}$ is $L_{r}$. The number of lags that go into the $x$ part of the polynomial $P(z, x)$ is $L_{p}$. The parameters $K_{z}$ and $K_{x}$ determine the degree of $P(z, x)$ and hence the nature of the innovation process $\left\{z_{t}\right\} . I_{z}$ and $I_{x}$ determine filters that suppress interactions when set to positive values.

Putting certain of the tuning parameters to zero implies sharp restrictions on the process $\left\{y_{t}\right\}$, the more interesting of which are given in Table 4 . 


\subsection{SNP fitting of the conditional density}

To determine the appropriate SNP specification, we follow a strategy similar to that of Gallant and Tauchen (1992a). The basic idea is to use model selection criteria jointly with a battery of specification tests on the conditional mean and variance. The outcome of this process yields the best fitting preferred model that the structural model of Section 2 must confront.

Table 2 shows the salient characteristics of the hierarchy of fitted SNP models. Each model is estimated over the basic data set comprised of 834 observations on $y_{t}$, with the first six observations reserved for forming lags, leaving a net of 828 observations. The table shows the settings of the tuning parameters, the number of parameters of the model, the value of the objective function, the values of the Schwarz, Hannan-Quinn, and Akaike model selection criteria (see Schwarz, 1978; Hannan, 1987), together with the $R^{2}$ 's for the diagnostics on the standardized residuals. The standardized residuals are the elements of $\left[\operatorname{var}_{t-1}\left(y_{t}\right)\right]^{-1 / 2}\left[y_{t}-\mathrm{E}_{t-1}\left(y_{t}\right)\right]$. The $R^{2}$ 's in the columns labeled 'mean' measure the predictability of the standardized residuals while those in the columns labeled 'variance' measure the predictability of the squares of the standardized residuals. Under the maintained hypothesis that the model of a particular row represents a correct specification, the underlying parent $R^{2}$ 's for that row are zero. Thus, small values of the $R^{2}$ 's indicate that a model is doing well in terms of capturing the conditional mean and variance functions.

The Schwarz criterion is $s_{n}(\hat{\theta})+\left(p_{\theta} / n\right) \log (n) / 2$, Hannan-Quinn is $s_{n}(\hat{\theta})+$ $\left(p_{\theta} / n\right) \log [\log (n)]$, and Akaike is $s_{n}(\hat{\theta})+\left(p_{\theta} / n\right)$, where $s_{n}(\theta)$ is the SNP optimization criterion and $p_{\theta}$ is the number of free parameters in the model. Each of these three criteria takes the form $s_{n}(\hat{\theta})+\left(p_{\theta} / n\right) c_{n}$, where $c_{n}$ is a penalty, and a smaller value of a criterion indicates a better fit. For samples as large as ours, the $c_{n}$ are such that the Schwarz criterion must select the smallest model and Akaike the largest, with Hannan-Quinn in between, though ties are possible. Asymptotic analysis (Hannan, 1987; Potscher, 1989) indicates that were the true model a stationary finitedimensional ARMA, then a criterion such that $c_{n} / n \rightarrow 0$ and $c_{n}>\log [\log (n)]$ for large enough $n$ will select the true ARMA model with probability one as $n \rightarrow \infty$. On the other hand, criteria such that $\lim _{\sup _{n \rightarrow \infty}} c_{n} / n>0$ will underfit and those such that $\lim \inf _{n \rightarrow \infty} c_{n} / \log [\log (n)]<1$ will overfit with positive probability. Thus, the Schwarz is a consistent criterion; the Hannan-Quinn defines a lower boundary on consistent criteria, while Akaike is certain to overfit for large enough $n$. For SNP estimation, these asymptotic results are only suggestive, because there is no claim that the true model is a finite-dimensional SNP, and there is always some bias associated with any selected finite-dimensional model. For our purposes, Schwarz might be expected to select too small of a model, a conjecture borne out in practice because it almost always selects a model that does poorly on specification tests (Gallant and Tauchen, 1992a). Indeed, Eastwood (1991) finds that criteria asymptotically equivalent to the Akaike are optimal in a mean-squared-error sense for SNP estimation of a nonlinear regression via a Fourier series. 
An immediate finding from Table 2 is the strong evidence for non-Gaussianity and conditional heteroskedasticity. The non-Gaussian ARCH models do substantially better on any of the three model selection criteria than do the preceding models, which are either VAR models or Gaussian ARCH models.

The model identified by the Schwarz criterion is the 304300 model. This same model is identified by the Hannan-Quinn criterion while a much larger model (414220) is identified by Akaike. The 304300 model has a lag length of 3 in the ARCH part, a quartic $\left(K_{z}=4\right)$ in the $z$ direction of the polynomial with all interactions suppressed $\left(I_{z}=3\right)$, and no other conditional heterogeneity. The diagnostic $R^{2}$ 's indicate that this model still leaves predictability in the standardized residuals and their squares, mainly in the interest rate residuals ( 7.4 percent) and in the conditional variances of the forward premium and the interest rate ( 9.8 percent and 6.7 percent, respectively), all of which are strongly statistically significant $R^{2}$ 's. This predictability suggests an increase in the size of the model is warranted.

The next-best model under the Schwarz criterion is the 304200 model, which does no better on the diagnostics, because this model includes no conditional heterogeneity beyond ARCH. The next-best under the Hannan-Quinn criterion is 314310 , which incorporates additional conditional heterogeneity beyond ARCH via $L_{p}=1$ and $K_{x}=1$. For this model, all of the diagnostic $R^{2}$ 's are 5 percent or less. While some $R^{2}$ 's remain statistically significant, all seem small from a practical perspective. Since expansion of the model beyond this point generates only marginal further reductions in the $R^{2}$ 's, we thus take the 314310 model as the best-fitting preferred model. Because the SNP specification does not impose stationarity directly on the fit, we performed one final check by plotting simulated realizations on $\left\{y_{t}\right\}$ and the one-step-ahead conditional moments from this model. We saw no evidence for explosive behavior or other forms of nonstationarity or anomalies.

To sum up, we find strong evidence that a model accommodating nonnormalities, $\mathrm{ARCH}$, plus other nonlinearities is needed to capture the full dynamics of the weekly German-U.S. currency market, 1975-90. The preferred SNP model from our specification search has a quartic in the error density whose coefficients depend linearly upon one lag of each variable. This model is defined by 96 parameters estimated on 828 trivariate observations, for a saturation ratio of 25.8 observations per parameter.

We now turn to estimation of the structural parameter $\lambda_{0}$ using this preferred estimate of the law of motion of $y_{t}$ as the standard of comparison.

\section{Empirical results}

We now apply the nonparametric structural estimator to both the transactions cost model and the cash-in-advance model described in Section 2 above. The SNP model defining the standard of comparison is the best-fitting model (314310) obtained in the previous section. This SNP model incorporates nonnormality and conditional 
heterogeneity above the ARCH. With 96 free parameters, the model thereby defines 96 indicators in the scoring function.

The minimization of the GMM criterion function is carried out using a simplex method of minimization developed by Nelder and Mead and provided in the GQOPT numerical optimization package. We have found this method to work much better for our problem than more traditional optimizers that use local gradient search methods. Our experience with such optimizers in this context has been that they are computationally expensive because of the large number of function calls needed to determine numerical derivatives and that they often settle at local optima far from the solutions obtained with the simplex method.

The estimation is computationally demanding, primarily because the simplex method requires so many function evaluations and each function evaluation entails recomputing a simulated realization of the equilibrium model. On a SUN SPARCstation 2, it takes about 1.5 minutes to compute the realization and evaluate the function. Typically, one run of the optimization entails several thousand function evaluations, so the run can extend across several days. Because the surface of the objective function is irregular, the optimizer must be restarted from several different start values, so the fitting can take weeks. Still, with recent improvement in hardware (e.g., the SUN SPARCstation 10 benchmarks to five to ten times faster than the SUN SPARCstation 2, and some HP machines are faster yet), the estimator for this model will soon be practicable on an overnight basis on a desktop workstation. We emphasize that the high computational cost is due almost exclusively to the iterative calculations needed to compute the equilibrium of the model, and not to any intrinsic properties of the estimator itself. In other on-going applications where the simulations are cheap to compute, the estimator is computable in an hour or two on a good workstation.

\subsection{Transaction cost model}

Tables 5 and 6 show the parameter estimates obtained from estimating $\lambda_{0}$ for the transaction cost model with $L_{c}=1$ and $L_{c}=2$, respectively. The economic model contains 37 or 39 free parameters depending upon whether $L_{c}$ equals 1 or 2 , so the asymptotic $\chi^{2}$ has either 59 or 57 degrees of freedom depending upon $L_{c}$.

As seen from these two tables, the chi-square statistics indicate the model is sharply rejected at either lag length. These chi-square statistics are not attenuated to account for the size of the simulation relative to the observed data set. Such an adjustment would be on the order of $(834 / 1000)$, which could hardly overturn the sharp rejections. Thus, economic inferences based on the fitted parameters must be interpreted with circumspection. With that said, it is still interesting to note that the curvature parameter $\gamma$ is estimated to be either 1.47 or 1.71 indicating a modest amount of risk aversion. When $L_{c}=1$, the $\kappa$ parameters are about 0.50 , suggesting strong local durability. When $L_{c}$ is increased to 2, however, there is an indication of habit persistence (see Heaton, 1993). Simulation evidence reported in Bekaert 
Table 5

Optimization results, $L_{c}=1$; transaction cost model

\begin{tabular}{|c|c|}
\hline$\beta$ & 0.9981 \\
\hline$\gamma$ & 1.4733 \\
\hline$\delta$ & 0.5663 \\
\hline$\psi_{0}$ & 0.0008380 \\
\hline$\alpha$ & 3.0433 \\
\hline$\kappa_{11}$ & 0.5261 \\
\hline \multicolumn{2}{|l|}{$\kappa_{12}$} \\
\hline$\kappa_{21}$ & 0.5302 \\
\hline \multicolumn{2}{|l|}{$k_{22}$} \\
\hline$a_{01}$ & $-0.3084 \mathrm{E}-04$ \\
\hline$a_{02}$ & $0.3519 \mathrm{E}-03$ \\
\hline$a_{03}$ & $-0.3439 \mathrm{E}-03$ \\
\hline$a_{04}$ & $0.1907 \mathrm{E}-02$ \\
\hline$A_{11}$ & $0.9405 \mathrm{E}+00$ \\
\hline$A_{21}$ & $0.4726 \mathrm{E}+00$ \\
\hline$A_{31}$ & $0.3514 \mathrm{E}+00$ \\
\hline$A_{41}$ & $-0.4827 \mathrm{E}+00$ \\
\hline$A_{12}$ & $0.9325 \mathrm{E}-03$ \\
\hline$A_{22}$ & $0.7338 \mathrm{E}+00$ \\
\hline$A_{32}$ & $-0.1669 \mathrm{E}-01$ \\
\hline$A_{42}$ & $-0.6727 \mathrm{E}-01$ \\
\hline$A_{13}$ & $0.2302 \mathrm{E}-01$ \\
\hline$A_{23}$ & $-0.7123 \mathrm{E}-02$ \\
\hline$A_{33}$ & $-0.3447 \mathrm{E}+00$ \\
\hline$A_{43}$ & $-0.3868 \mathrm{E}+00$ \\
\hline$A_{14}$ & $0.4588 \mathrm{E}-02$ \\
\hline$A_{24}$ & $-0.6143 \mathrm{E}-01$ \\
\hline$A_{34}$ & $-0.4392 \mathrm{E}+00$ \\
\hline$A_{44}$ & $0.2995 \mathrm{E}+00$ \\
\hline$H_{11}$ & $0.8797 \mathrm{E}-05$ \\
\hline$H_{12}$ & $0.8727 \mathrm{E}-05$ \\
\hline $\mathrm{H}_{22}$ & $0.2039 \mathrm{E}-03$ \\
\hline$H_{13}$ & $0.2253 E-05$ \\
\hline $\mathrm{H}_{23}$ & $0.1224 \mathrm{E}-02$ \\
\hline $\mathrm{H}_{33}$ & $0.1011 \mathrm{E}-03$ \\
\hline$H_{14}$ & $0.5149 \mathrm{E}-05$ \\
\hline $\mathrm{H}_{24}$ & $-0.8768 \mathrm{E}-03$ \\
\hline $\mathrm{H}_{34}$ & 0.6344 E-04 \\
\hline$H_{44}$ & $0.9568 \mathrm{E}-04$ \\
\hline$\tilde{s}_{n}(\hat{\lambda})$ & $\chi^{2}(59)=478.8875$ \\
\hline & $p$-value $<0.0001$ \\
\hline
\end{tabular}

$\ln \left(S_{t}\right)=a_{0}+A \ln \left(S_{t-1}\right)+u_{t}$, where $S_{t}=$ $\left(\mathrm{d} M_{1 t}, \mathrm{~d} M_{2 t}, \mathrm{~d} w_{1 t}, \mathrm{~d} w_{2 t}\right)^{\prime}, a_{0}$ is $4 \times 1, A$ is $4 \times 4, u_{t}$ is a $4 \times 1$ iid $\mathrm{N}(0, \Omega)$ process, and $\Omega=H H^{\prime}$ with $H$ taken as upper triangular.
Table 6

Optimization results, $L_{c}=2$; transaction cost model

\begin{tabular}{ll}
\hline$\beta$ & 0.9969 \\
$\gamma$ & 1.7010 \\
$\delta$ & 0.7637 \\
$\psi_{0}$ & 0.0004704
\end{tabular}

$\alpha \quad 2.1928$

$\kappa_{11} \quad 0.3265$

$\kappa_{12}-0.7756$

$\kappa_{21} \quad 0.1027$

$\kappa_{22}-0.4184$

$a_{01} \quad-0.2019 \mathrm{E}-03$

$a_{02} \quad-0.7647 \mathrm{E}-04$

$a_{03} \quad 0.6844 \mathrm{E}-03$

$a_{04} \quad 0.2673 \mathrm{E}-03$

$A_{11} \quad 0.9167 \mathrm{E}+00$

$A_{21} \quad 0.3483 \mathrm{E}+00$

$A_{31} \quad-0.3035 \mathrm{E}+00$

$A_{41} \quad-0.1198 \mathrm{E}+00$

$A_{12} \quad 0.5589 \mathrm{E}-02$

$A_{22} \quad 0.7778 \mathrm{E}+00$

$A_{32} \quad-0.5233 \mathrm{E}-01$

$A_{42} \quad-0.6817 \mathrm{E}-01$

$A_{13} \quad-0.2966 \mathrm{E}-01$

$A_{23} \quad 0.3442 \mathrm{E}+00$

$A_{33} \quad 0.5467 \mathrm{E}-01$

$A_{43} \quad 0.3872 \mathrm{E}+00$

$A_{14} \quad 0.3035 \mathrm{E}-01$

$A_{24} \quad 0.1108 \mathrm{E}+00$

$A_{34} \quad-0.8315 \mathrm{E}+00$

$A_{44} \quad 0.4409 \mathrm{E}+00$

$H_{11} \quad 0.9499 \mathrm{E}-05$

$\mathrm{H}_{12} \quad 0.4720 \mathrm{E}-05$

$\mathrm{H}_{22} \quad 0.3327 \mathrm{E}-03$

$H_{13} \quad 0.2013 \mathrm{E}-05$

$\mathrm{H}_{23} \quad 0.1458 \mathrm{E}-02$

$\mathrm{H}_{33} \quad 0.9501 \mathrm{E}-04$

$H_{14} \quad 0.6102 \mathrm{E}-05$

$\mathrm{H}_{24} \quad-0.2806 \mathrm{E}-03$

$\mathrm{H}_{34} \quad 0.1242 \mathrm{E}-03$

$\mathrm{H}_{44} \quad 0.1231 \mathrm{E}-03$

$\tilde{s}_{n}(\hat{\lambda}) \quad \chi^{2}(57)=333.5919$ $p$-value $<0.0001$

$\ln \left(S_{t}\right)=a_{0}+A \ln \left(S_{t-1}\right)+u_{t}$, where $S_{t}=$ $\left(\mathrm{d} M_{1 t}, \mathrm{~d} M_{2 t}, \mathrm{~d} w_{1 t}, \mathrm{~d} w_{2 t}\right)^{\prime}, a_{0}$ is $4 \times 1, A$ is $4 \times 4, u_{t}$ is a $4 \times 1$ iid $\mathrm{N}(0, \Omega)$ process, and $\Omega=H H^{\prime}$ with $H$ taken as upper triangular. 


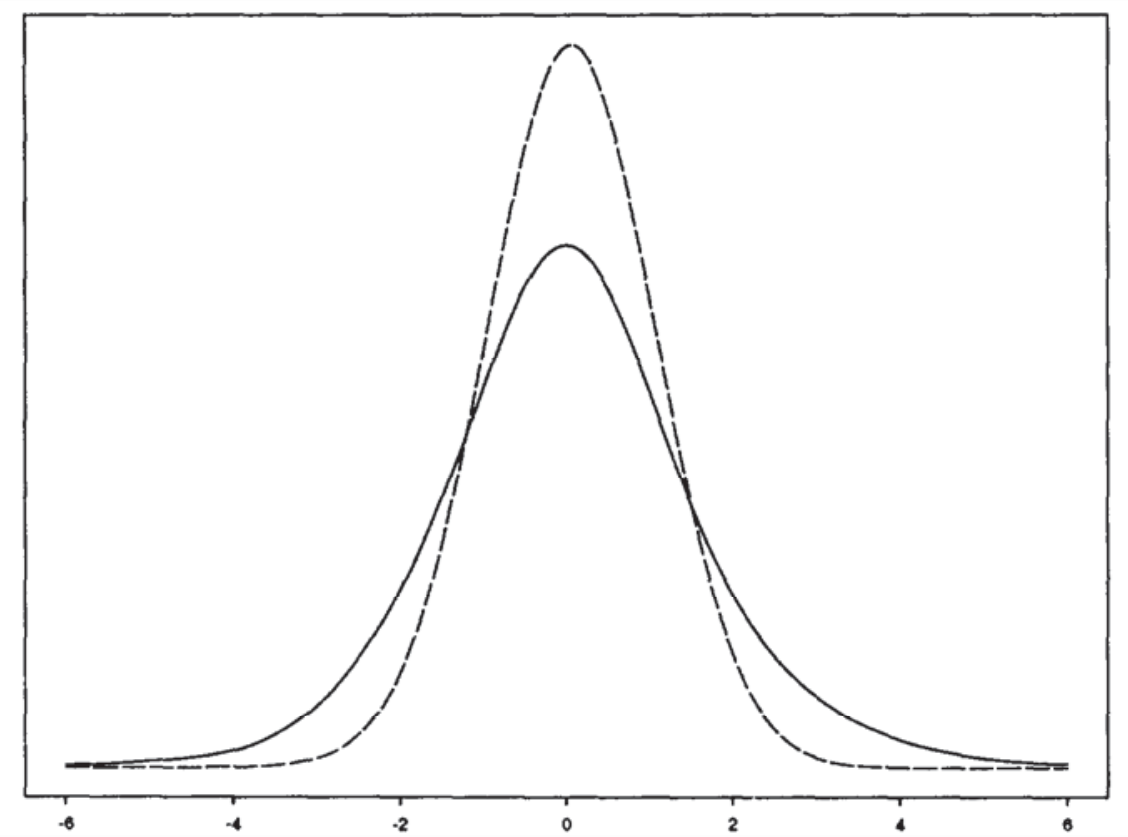

Fig. 1. Kernel estimates of the unconditional density of differenced spot $\log ($ SUS/DM) times 100 . Solid line is actual data. Dashed line is simulated data from the transaction cost model with $L_{c}=2$.

(1991) suggests that this pattern is very important if a monetary model is to match the autocorrelation structure of the forward premium. The estimates of $\alpha$ indicate that the transaction cost technology is highly nonlinear. Also, the estimates of $\alpha$ and $\phi_{0}$ together with the estimated velocity processes suggest that approximately one twentieth of 1 percent of the endowment is absorbed in transaction costs each week, a not implausible point estimate. The point estimates of the parameters of the law of motion for strictly exogenous vector $S_{t}$ suggest complex patterns of feedback between the series with strong persistence in the weekly money growth rates.

Figs. 1 through 3 show the unconditional distributions of $y_{1 t}, y_{2 t}$, and $y_{3 t}$, estimated by applying a kernel method to the observed data and to the simulated data from the model with $L_{c}=2$; the figures are similar for $L_{c}=1$ and so are not shown. The solid lines in the figures correspond to the distributions of the observed data and the dashed lines to the distributions of the simulated data. The figures suggest that the model does a good job on fitting the means but is understating the volatility, though less severely for the spot growth rate. The Gaussian shape of the distributions for the simulated data, and in particular the symmetry and moderate tail behavior, suggests that the model does not generate nonlinearity to the same degree as present in the observed data. We explored the effects of using a $t$-distribution with six degrees of freedom as the error density of the latent driving processes. This 
Table 7

Autocorrelation functions

\begin{tabular}{|c|c|c|c|c|c|c|}
\hline \multicolumn{7}{|c|}{$\begin{array}{l}\text { Autocorretation functions: } \operatorname{corr}\left(y_{i, t}, y_{j, t-k}\right) \\
\qquad \underline{i, j}\end{array}$} \\
\hline$k$ & 1,1 & 1,2 & 2,2 & 1.3 & 2,3 & 3,3 \\
\hline \multicolumn{7}{|c|}{ Actual data } \\
\hline-5 & -0.061 & -0.120 & 0.789 & -0.140 & 0.641 & 0.924 \\
\hline-4 & 0.045 & -0.118 & 0.840 & -0.142 & 0.661 & 0.943 \\
\hline-3 & 0.016 & -0.127 & 0.886 & -0.137 & 0.676 & 0.956 \\
\hline-2 & 0.034 & -0.127 & 0.927 & -0.138 & 0.689 & 0.970 \\
\hline-1 & 0.021 & -0.145 & 0.965 & -0.136 & 0.698 & 0.985 \\
\hline 0 & 1.000 & -0.128 & 1.000 & -0.126 & 0.702 & 1.000 \\
\hline 1 & 0.021 & -0.105 & 0.965 & -0.096 & 0.690 & 0.985 \\
\hline 2 & 0.034 & -0.107 & 0.927 & -0.077 & 0.670 & 0.970 \\
\hline 3 & 0.016 & -0.094 & 0.886 & -0.082 & 0.645 & 0.956 \\
\hline 4 & 0.045 & -0.056 & 0.840 & -0.070 & 0.621 & 0.943 \\
\hline 5 & -0.061 & -0.050 & 0.789 & -0.063 & 0.595 & 0.924 \\
\hline \multicolumn{7}{|c|}{ Simulated data - Transaction cost model } \\
\hline-5 & 0.022 & -0.080 & 0.339 & 0.018 & -0.042 & 0.088 \\
\hline-4 & 0.004 & -0.147 & 0.379 & 0.030 & -0.079 & 0.121 \\
\hline-3 & -0.018 & -0.191 & 0.433 & 0.071 & -0.057 & 0.101 \\
\hline-2 & 0.012 & -0.159 & 0.524 & 0.136 & -0.038 & 0.125 \\
\hline-1 & 0.015 & 0.053 & 0.696 & 0.130 & -0.003 & 0.268 \\
\hline 0 & 1.000 & 0.004 & 1.000 & -0.100 & 0.028 & 1.000 \\
\hline 1 & 0.015 & 0.004 & 0.696 & 0.029 & 0.024 & 0.268 \\
\hline 2 & 0.012 & -0.008 & 0.524 & -0.018 & 0.046 & 0.125 \\
\hline 3 & -0.018 & 0.040 & 0.433 & -0.022 & 0.006 & 0.101 \\
\hline 4 & 0.004 & 0.030 & 0.379 & 0.008 & 0.016 & 0.121 \\
\hline 5 & 0.022 & 0.028 & 0.339 & 0.018 & 0.007 & 0.088 \\
\hline \multicolumn{7}{|c|}{ Squared VAR residuals of actual data } \\
\hline-5 & 0.076 & 0.015 & 0.195 & 0.055 & 0.095 & 0.121 \\
\hline-4 & 0.056 & 0.064 & 0.342 & 0.643 & 0.227 & 0.174 \\
\hline-3 & 0.147 & 0.033 & 0.406 & 0.083 & 0.197 & 0.114 \\
\hline-2 & 0.077 & 0.039 & 0.157 & 0.065 & 0.214 & 0.196 \\
\hline-1 & 0.135 & 0.118 & 0.247 & 0.033 & 0.207 & 0.316 \\
\hline 0 & 1.000 & 0.169 & 1.000 & 0.044 & 0.394 & 1.000 \\
\hline l & 0.135 & -0.011 & 0.247 & 0.020 & 0.167 & 0.316 \\
\hline 2 & 0.077 & -0.003 & 0.157 & 0.008 & 0.166 & 0.196 \\
\hline 3 & 0.147 & 0.146 & 0.406 & 0.022 & 0.353 & 0.114 \\
\hline 4 & 0.056 & 0.022 & 0.342 & 0.043 & 0.241 & 0.174 \\
\hline 5 & 0.076 & 0.014 & 0.195 & 0.034 & 0.124 & 0.121 \\
\hline \multicolumn{7}{|c|}{ Squared VAR residuals of simulated data } \\
\hline-5 & 0.049 & 0.061 & -0.082 & 0.069 & 0.052 & 0.064 \\
\hline-4 & 0.069 & 0.071 & 0.080 & 0.047 & 0.039 & 0.054 \\
\hline-3 & 0.066 & 0.143 & 0.025 & 0.088 & 0.011 & 0.035 \\
\hline-2 & 0.069 & 0.134 & 0.117 & 0.170 & 0.053 & -0.021 \\
\hline-1 & 0.048 & 0.112 & 0.061 & 0.119 & 0.027 & 0.018 \\
\hline 0 & 1.000 & 0.065 & 1.000 & 0.053 & 0.111 & 1.000 \\
\hline 1 & 0.048 & 0.020 & 0.061 & 0.035 & 0.111 & 0.018 \\
\hline 2 & 0.069 & 0.069 & 0.117 & 0.113 & 0.084 & -0.021 \\
\hline 3 & 0.066 & 0.041 & 0.025 & -0.005 & 0.107 & 0.035 \\
\hline 4 & 0.069 & 0.032 & 0.080 & -0.027 & 0.089 & 0.054 \\
\hline 5 & 0.049 & 0.023 & -0.082 & 0.007 & 0.030 & 0.064 \\
\hline
\end{tabular}




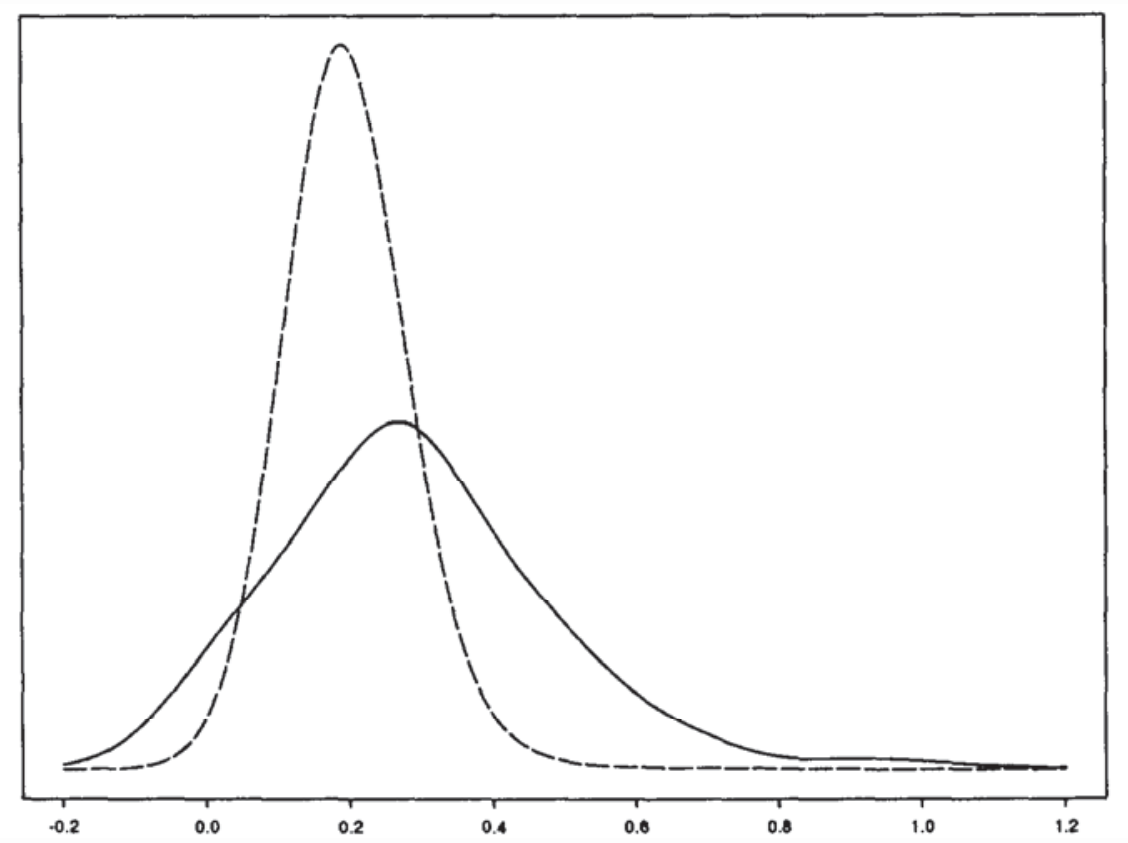

Fig. 2. Kernel estimates of the unconditional density of 30 day forward $\log$ (\$US/DM) less spot $\log ($ SUS/DM) times 100 . Solid line is actual data. Dashed line is simulated data from the transaction cost model with $L_{c}=2$.

distribution has much thicker tails than the Gaussian, and the model is able to generate more leptokurtosis in the currency data. But also, the model does less well in fitting some key autocorrelations, so it does less well overall in terms of the value of the chi-square statistic.

The top and middle portions of Table 7 show the autocorrelation function of the observed data $\left\{y_{t}\right\}$ and the simulated data $\left\{y_{\tau}^{\hat{\hat{k}}}\right\}$ from the model with $L_{c}=2$. Though our nonparametric structural estimator attempts to fit much more than linear dependence in the series, these autocorrelations do show where the model is having success in fitting the data. Similar to the observed data, the model is generating little persistence in $y_{1 t}$ and positive persistence in $y_{2 t}$, though the latter feature is understated in the simulated data. Likewise, both the observed and simulated data display small cross correlations between $y_{1 t}$ and $y_{2 t}$ and between $y_{1 t}$ and $y_{3 t}$. The major shortcomings in the fit are the lack of very strong persistence in $y_{3 t}$ and the absence of strong cross correlations between $y_{2 t}$ and $y_{3 t}$. It is interesting to note, however, that some values for $\lambda$ that the optimizer tried did generate simulated data with these latter two properties, but the optimizer ultimately moved away from that region of the parameter space. Thus the model seems capable of generating strong persistence in $y_{3 t}$ and strong cross-correlations between $y_{2 t}$ and $y_{3 t}$, but we have 


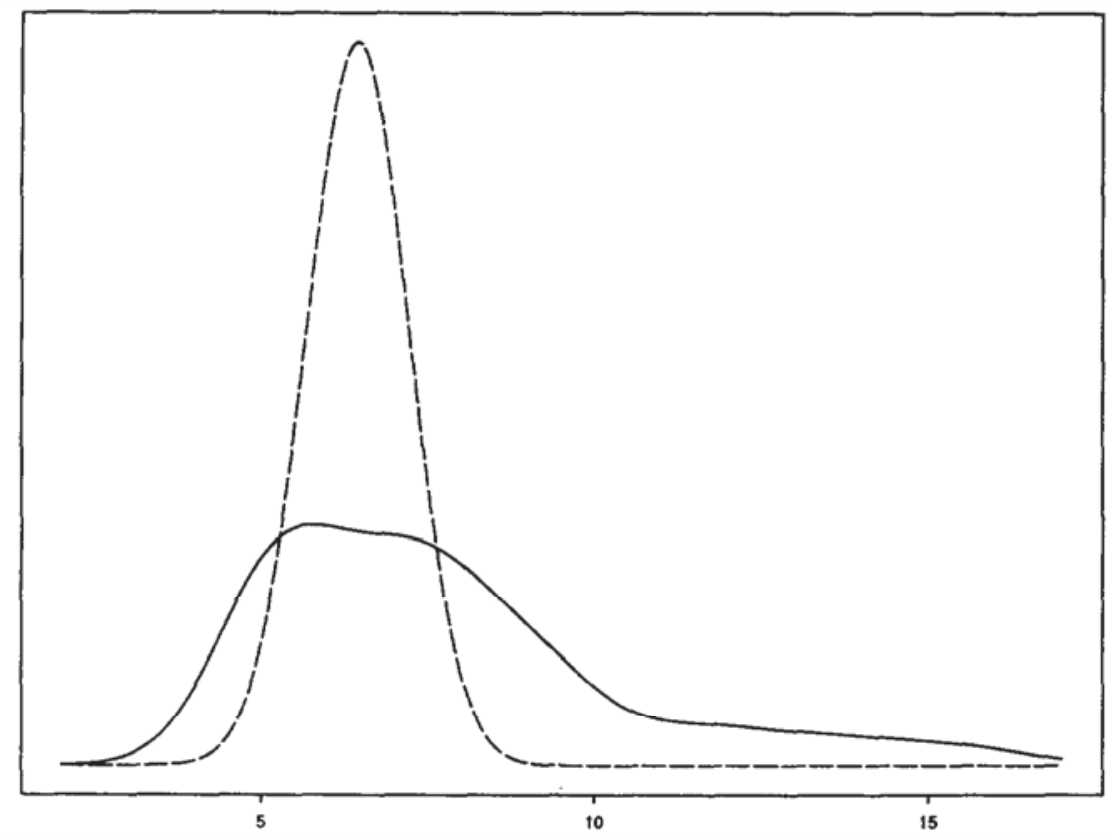

Fig. 3. Kernel estimates of the unconditional density of 30 day SUS interest rate. Solid line is actual data. Dashed line is simulated data from the transaction cost model with $L_{c}=2$.

not yet seen it do so in conjunction with matching the other important properties in the observed data.

The bottom portion of Table 7 shows the autocorrelation function of squared VAR(3) residuals fitted to observed and simulated data. This table suggests that the model is generating only some of the conditional heteroskedasticity present in the observed data, and occasionally the patterns of heteroskedasticity are substantially different between the observed and simulated data.

We also computed in the simulated data the $R^{2}$ 's from regressions of $s_{t+4}-f_{t}^{4}$ on various lags of $f_{t}^{4}-s_{t}$ and other state variables. We obtain $s_{t+4}-f_{t}^{4}$ from the simulated data by computing $s_{t+4}-f_{t}^{4}=y_{1, t+1}+\cdots+y_{1, t+4}-y_{2 t}$. Note that $s_{t+4}-f_{t}^{4}$ and $f_{t}^{4}-s_{t}$ correspond to the observed series $100 *\left(\log \left[S P O T_{t+4}\right]-\right.$ $\log \left[\right.$ FORWARD $\left.\left._{t}\right]\right)$ and $100 *\left(\log \left[F O R W A R D_{t}^{4}\right]-\log \left[\right.\right.$ SPOT $\left.\left._{t}\right]\right)$, which were used in the test of unbiased forward rates reported in Table 3 . These regressions typically had very low $R^{2}$ 's (less that 0.01 ), suggesting that the model does not generate a risk premia that is linearly predictable to the extent indicated in Table 3.

Good data on endowments and money growth are not available weekly but are available monthly. We compared the properties of simulated data on the exogenous processes at the monthly frequency to observed monthly U.S. data. Simulated monthly series are appropriately formed four-period aggregates of the simulated 
weekly processes; observed monthly data are from Citibase for the period 1959.011991.06. Consistent with the interpretation of the model, the endowment process for country 1 corresponds to the observed U.S. aggregate consumption of nondurables and services; money growth corresponds to an aggregate of U.S. money growth (M1 or M2). We find that the fitted model generally understates the mean and variance of endowment growth and money growth. For the endowment process, the mean and standard deviation of monthly growth rates in the simulation are 0.0011 and 0.00035 , versus 0.055 and 0.0077 , in the observed data; for the money process, the mean and standard deviation of monthly growth rates in the simulation are -0.086 and 0.0002 , versus 0.046 and 0.0047 for M1 and 0.063 and 0.0033 for M2. Also, the fitted model implies positive autocorrelation at lag one in the endowment process, 0.39 , versus -0.27 in the observed data. On the other hand, the model does better in terms of matching the persistence in money growth rates. The first three autocorrelations of simulated monthly money growth rates are $0.79,0.58,0.44$, versus 0.36 , $0.18,0.25$ for $\mathrm{M} 1$ and $0.66,0.42,0.36$ for M2.

Despite the mild success with the persistence of money growth rates, the model generally fares poorly on this comparison. This outcome is not surprising, given that the model is fitted using only weekly asset returns, without any data on physical quantities such as endowments and money supplies, and it does not do well under the usual statistical measure of within-sample fit. Thus, it does not do very well when extrapolated outside the sample.

The estimation procedure could be generalized to bring in the additional information from data on exogenous processes at a higher level of temporal aggregation. Two routes are possible, though each has pitfalls. One would be to use the temporally aggregated data to estimate the implied law of motion of the driving process (at the high frequency), and then condition on that estimate in a second round estimation on the high-frequency data. This route entails the typical problems with twostep estimators, especially with defining the second-step objective function so its optimized value is asymptotically chi-square. A second strategy would be to define an additional moment criterion (observed versus predicted under the simulation), which would be concatenated with the criterion used on this paper and then a single optimization performed. A nonparametric estimate of the conditional density of the temporally aggregated data could be used to define the criterion. The complicating factor would be to develop an estimate of the covariance of the criteria across levels of temporal aggregation, which is essential to estimating a GMM weight matrix that makes the minimized value of the objective function asymptotically chi-square.

\subsection{Cash-in-advance model}

Table 8 shows the estimated parameters obtained by estimating the cashin-advance model with $L_{c}=2$ in the same manner as done for the transaction cost model. Comparison with Table 6 shows some of the contrasts between the models. With the fitted $\gamma$ being near zero, the cash-in-advance specification shows much 
Table 8

Optimization results, $L_{c}=2$ : cash-in-advance model

\begin{tabular}{|c|c|}
\hline$\beta$ & 0.9984 \\
\hline$\gamma$ & 0.1149 \\
\hline$\delta$ & 0.2448 \\
\hline$\kappa_{11}$ & 0.9371 \\
\hline$\kappa_{12}$ & 0.5930 \\
\hline$\kappa_{21}$ & -0.2524 \\
\hline$\kappa_{22}$ & 0.0792 \\
\hline$a_{01}$ & $0.2604 \mathrm{E}-02$ \\
\hline$a_{02}$ & $0.7498 \mathrm{E}-03$ \\
\hline$a_{03}$ & $-0.1133 \mathrm{E}-01$ \\
\hline$a_{04}$ & $0.6894 \mathrm{E}-02$ \\
\hline$A_{11}$ & $-0.1737 \mathrm{E}-01$ \\
\hline$A_{21}$ & $-0.7587 \mathrm{E}-01$ \\
\hline$A_{31}$ & $0.4387 \mathrm{E}+00$ \\
\hline$A_{41}$ & $-0.2411 E+00$ \\
\hline$A_{12}$ & $0.1011 \mathrm{E}+00$ \\
\hline$A_{22}$ & $0.1750 \mathrm{E}+00$ \\
\hline$A_{32}$ & $0.4019 E+01$ \\
\hline$A_{42}$ & $-0.9009 E+00$ \\
\hline$A_{13}$ & 0.1927 E -01 \\
\hline$A_{23}$ & $0.1127 \mathrm{E}+00$ \\
\hline$A_{33}$ & $-0.9140 E+00$ \\
\hline$A_{43}$ & $0.3750 \mathrm{E}+00$ \\
\hline$A_{14}$ & $0.1232 \mathrm{E}+00$ \\
\hline$A_{24}$ & $0.3608 \mathrm{E}+00$ \\
\hline$A_{34}$ & $-0.2658 \mathrm{E}+01$ \\
\hline$A_{44}$ & $0.1065 \mathrm{E}+01$ \\
\hline$H_{11}$ & $0.7128 \mathrm{E}-02$ \\
\hline$H_{12}$ & $-0.4767 \mathrm{E}-02$ \\
\hline $\mathrm{H}_{22}$ & $0.8531 \mathrm{E}-03$ \\
\hline$H_{13}$ & $-0.4225 \mathrm{E}-02$ \\
\hline $\mathrm{H}_{23}$ & $0.9151 \mathrm{E}-03$ \\
\hline$H_{33}$ & $-0.4426 \mathrm{E}-03$ \\
\hline$H_{14}$ & $-0.4787 \mathrm{E}-02$ \\
\hline $\mathrm{H}_{24}$ & $0.1925 \mathrm{E}-02$ \\
\hline $\mathrm{H}_{34}$ & $0.2502 \mathrm{E}-02$ \\
\hline$H_{44}$ & $0.7435 E-03$ \\
\hline \multirow[t]{2}{*}{$\tilde{s}_{n}(\hat{\lambda})$} & $x^{2}(59)=362.02637$ \\
\hline & $p$-value $<0.0001$ \\
\hline
\end{tabular}

$\ln \left(S_{t}\right)=a_{0}+A \ln \left(S_{t-1}\right)+u_{t}$, where $S_{t}=\left(\mathrm{d} M_{1 t}, \mathrm{~d} M_{2 t}, \mathrm{~d} w_{1 t}^{\prime}, \mathrm{d}_{w_{2 t}}\right)^{\prime}, a_{0}$ is $4 \times 1, A$ is $4 \times 4, u_{t}$ is a $4 \times 1$ iid $\mathrm{N}(0, \Omega)$ process, and $\Omega=H H^{\prime}$ with $H$ taken as upper triangular.

less curvature in utility than does the transaction cost specification. With $\delta$ estimated well below 0.50 , the cash-in-advance specification places substantially less weight on country 1 , whereas the transaction cost specification places much weight more on country 1 . As with the transaction cost specification, the cash-in-advance 


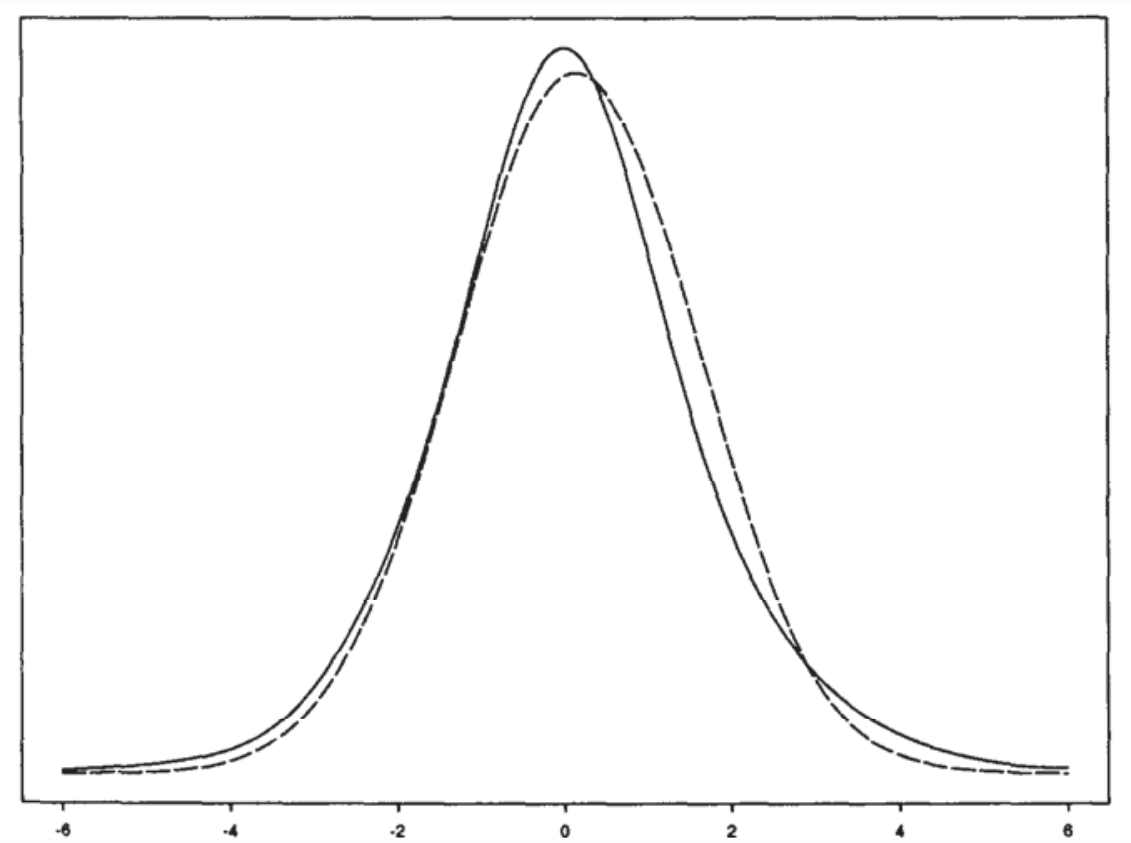

Fig. 4. Kernel estimates of the unconditional density of differenced spot $\log ($ SUS/DM) times 100 . Solid line is actual data. Dashed line is simulated data from the cash-in-advance model with $L_{c}=2$.

specification also detects for country 1 short-term durability and longer-term habit persistence as found with the transaction cost specification. At the same time, the cash-in-advance specification only finds habit persistence for country 2 .

Figs. 4 through 6 show the unconditional distributions of $y_{1 t}, y_{2 t}$, and $y_{3 t}$ implied by the fitted cash-in-advance model together with those of the observed data. Fig. 4 indicates that the cash-in-advance specification does exceptionally well in fitting the shape characteristics of the spot exchange rate distribution. For this specification, it turns out that the spot growth rate is predicted to be nearly serially uncorrelated as well. Thus, a fit to first and second moments of the exchange rate alone, instead of to the entire distribution of the data set as undertaken here, might have yielded substantially less evidence against the model.

Comparison of Figs. 5 and 6 with Figs. 2 and 3 suggests that the cash-in-advance specification, however, performs less well than the transaction cost specification in terms of fitting the forward premium and the interest rate series. In particular, the cash-in-advance model overpredicts the location of both distributions and underpredicts the scale. Also, the cash-in-advance specification does seem to put in some right skewness in the interest rate distribution, which seems completely absent in that implied by the transaction cost model (Fig. 3). 


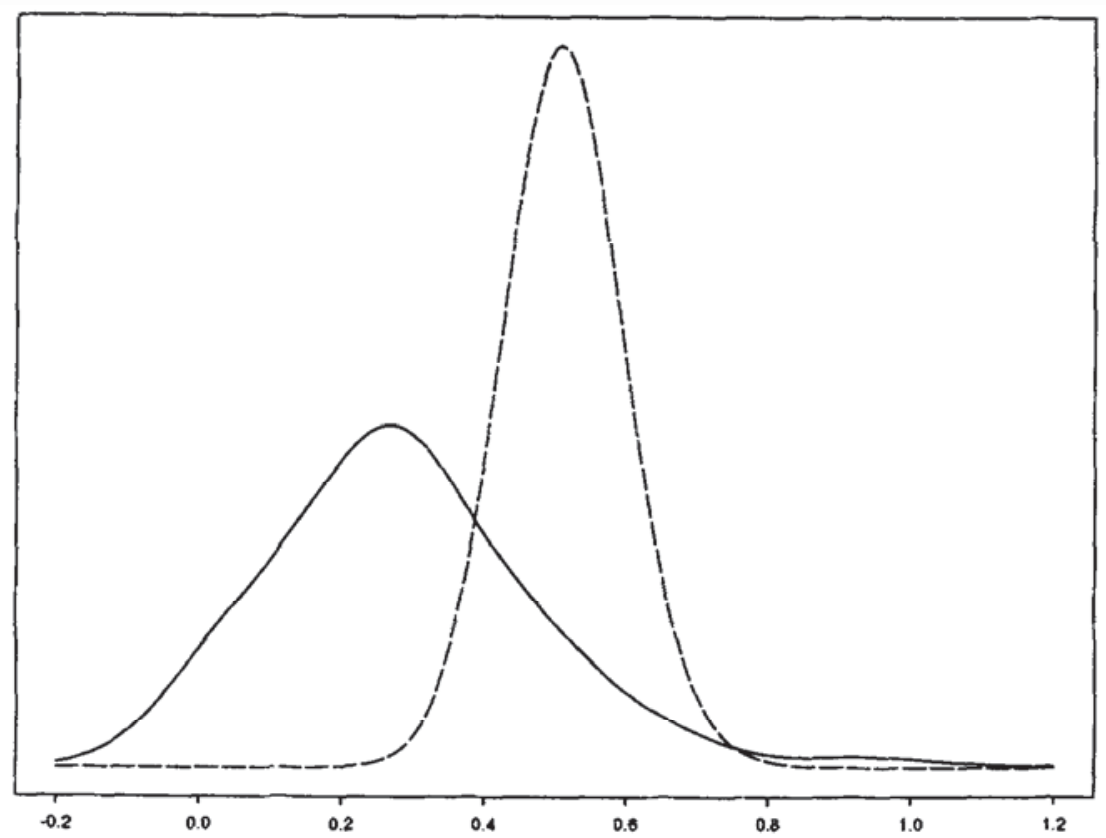

Fig. 5. Kernel estimates of the unconditional density of 30 day forward $\log$ (\$US/DM) less spot $\log (\$ U S / D M)$ times 100 . Solid line is actual data. Dashed line is simulated data from the cash-in-advance model with $L_{c}=2$.

\section{Conclusion}

In summary, the transaction cost model as currently fitted produces reasonable parameter values and does well in capturing first-moment characteristics of the data and some of the serial correlation structure. At the same time, though, it does poorly in matching the dynamic behavior of short-term interest rates, $y_{3 t}$, and in accommodating the higher-order structure of the data, particularly the non-Gaussian character of the marginal distributions and the stochastic structure of the volatility. For the most part, the cash-in-advance specification suffers the same difficulties.

We view the outcome as quite encouraging in terms of demonstrating the practicability of nonparametric structural estimation. The approach forces the economic model to confront the full nonparametric estimate of the law of motion of data and thereby face all empirically relevant aspects of the data.

This strategy demands much of the model. In particular it excludes the possibility of isolating a few selected dimensions along which the model does well while ignoring all of the others. This feature of the approach perhaps explains why the omnibus tests reject both the transaction cost and cash-in-advance models so sharply. Though the equilibrium models can generate the high volatility observed in the spot exchange rate, which is an aspect of the data of great importance in the literature, 


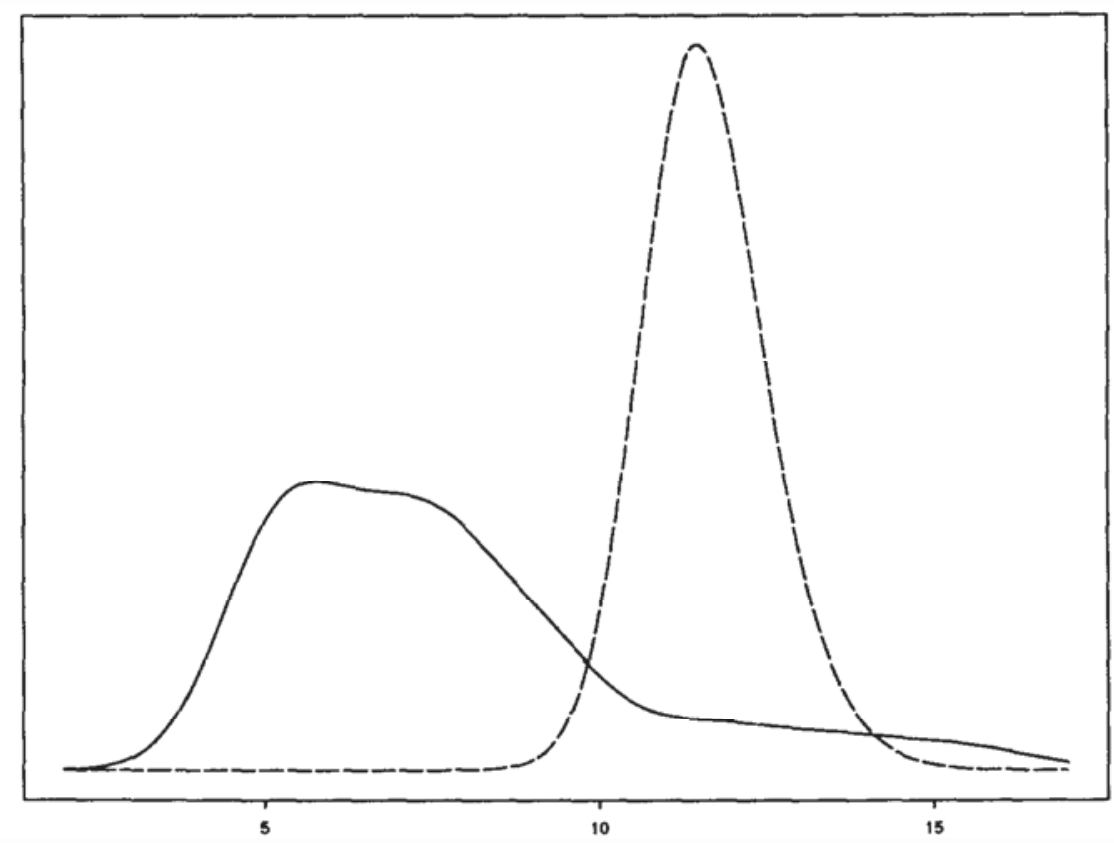

Fig. 6. Kernel estimates of the unconditional density of 30 day \$US interest rate. Solid line is actual data. Dashed line is simulated data from the cash-in-advance model with $L_{c}=2$.

the models come up short along other dimensions such as generating substantial stochastic volatility and nonnormality. One way to view the sharp rejections is to recognize that the estimation method optimally weights together many different indicators of fit in forming the objective function. Thus, the inability to reduce the objective function to insignificance reveals the empirical importance of those indicators along which the model fares less well. Overall, the finding suggests that an equilibrium model is too 'smooth' to produce realistic nonlinearity at the weekly frequency. Perhaps bubble models of money (Matsuyama, 1990; Boldrin and Woodford, 1990), which generate chaotic solution paths, could better accommodate the weekly data.

One reviewer's reaction to these final remarks was that few could expect a model of a representative agent solving an intertemporal optimization problem to face all empirically relevant aspects of the data. This reviewer felt the findings about an equilibrium model being too smooth left 'the reader alone in front of the central question of the usefulness of the structural model, if [one excludes] the possibility of isolating a few selected dimensions along which it does well and along which it could be used'. We agree that the procedure stretches structural modeling to its very limit. Our view, though, is that if a structural model is to be implemented and evaluated on statistical criteria, i.e., if one wants to take seriously statistical tests 
and inference, then the structural model has to face all empirically relevant aspects of the data. Statistical inference with a misspecified model is dubious practice at best (Gallant, 1987, pp. 217-240). If one eschews statistical criteria - as some are currently inclined to do - then one might develop a theory of inference that permits selection of a few isolated dimensions to the exclusion of all others. That theory of inference would inherently be nonstatistical, and, to our knowledge, it has yet to be worked out.

\section{References}

Andrews, Donald W.K., 1991, Asymptotic normality of series estimators for nonparametric and semiparametric models, Econometrica 59, 307-346.

Baillie, R.T., R.E. Lippens, and H.C. McMahon, 1983, Testing rational expectations and efficiency in the foreign exchange market, Econometrica 51, 553-564.

Bansal, Ravi, 1990, Can nonseparabilities explain exchange rate movements and risk premia?, Ph.D. dissertation (Carnegie Mellon University, Pittsburgh, PA).

Bansal, Ravi. A. Ronald Gallant, Robert Hussey, and George Tauchen, 1993, Computational aspects of nonparametric simulation estimation, in: David A. Belsley, ed., Computational techniques for econometrics and economic analysis (Kluwer Academic Publishers, Boston, MA) 3-22.

Baumol, William, 1952, The transactions demand for cash: an inventory approach, Quarterly Journal of Economics 66, 545-556.

Bekaert, Geert, 1991, The time variation of risk and return in foreign exchange markets: A general equilibrium perspective, Manuscript (Department of Economics, Northwestern University, Evanston, IL).

Bilson, J., 1981, The speculative efficiency hypothesis, Journal of Business 54, 435-452.

Boldrin. Michele and Michael Woodford, 1990. Equilibrium models displaying endogenous fluctuations and chaos: A survey, Journal of Monetary Economics 25, 189-222.

Bollerslev, Tim, Ray Chou, and Kenneth Kroner, 1992, ARCH modeling in finance: A review of the theory and empirical evidence, Journal of Econometrics 52, 5-59.

Cochrane, John and Lars Peter Hansen, 1992, Asset pricing explorations for macroeconomics, Working paper no. 4088 (NBER, Cambridge, MA).

Constantinides, George M., 1990, Habit formation: A resolution of the equity premium puzzle, Journal of Political Economy 98, 519-543.

Cumby, R. and M. Obstfeld, 1984, International interest rate and price level linkages under flexible exchange rates: A review of recent evidence, in: J. Bilson and R. Marston, eds., Exchange rate theory and practice (University of Chicago Press, Chicago, IL) 121-151.

Davidian, Marie and Raymond J. Carroll, 1987, Variance function estimation, Journal of the American Statistical Association 82, 1079-1091.

den Haan, Wouter J. and Albert Marcet, 1990, Solving the stochastic growth model by parameterizing expectations, Journal of Business and Economic Statistics 8, 31-34.

Dickey, David and Fuller, Wayne, 1979, Distribution of the estimators for autoregressive time series with a unit root, Journal of American Statistical Association 74, 427-431.

Dooley, M. and J. Shafer, 1983, Analysis of short-run exchange rate behavior: March 1973 to November 1981, in: D. Bigman and T. Taya, eds., Exchange rate and trade instability: Causes, consequences, and remedies (Ballinger, Cambridge, MA) 43-69.

Dornbusch, Rudiger and Jeffrey Frankel, 1987, The flexible exchange rate system: Experience and alternatives, Working paper no. 2464 (NBER, Cambridge, MA).

Duffie, Darrell and Kenneth J. Singleton, 1993, Simulated moments estimation of Markov models of asset prices, Econometrica 61, 929-952. 
Dunn, Kenneth and Kenneth J. Singleton, 1986, Modeling the term structure of interest rates under nonseparable utility and durability of goods, Journal of Financial Economics 17, 27-55.

Eastwood, Brian J., 1991, Asymptotic normality and consistency of seminonparametric regression estimators using an upwards $F$-test rule, Journal of Econometrics 48, 151-183.

Eichenbaum, Martin S. and Lars Peter Hansen, 1990, Estimating models with intertemporal substitution using aggregate time series data, Journal of Business and Economic Statistics 8, 53-70.

Eichenbaum, Martin, Lars Peter Hansen and Kenneth J. Singleton, 1988, A time series analysis of representative agent models and consumption and leisure choice under uncertainty, Quarterly Journal of Economics 103, 51-78.

Engle, Robert F., 1982, Autoregressive conditional heteroscedasticity with estimates of the variance of United Kingdom inflation, Econometrica 50, 987-1007.

Engle, Robert F. and Gloria Gonzales-Rivera, 1991, Semiparametric ARCH models, Journal of Business and Economic Statistics 9, 345-360.

Feenstra, R.C., 1986, Functional equivalence between liquidity costs and the utility of money, Journal of Monetary Economics 17, 271-291.

Frankel, Jeffrey, 1984, Tests of monetary and portfolio-balance models of exchange rate determination, in: J. Bilson and R. Marston, eds., Exchange rate theory and practice (University of Chicago Press. Chicago, IL) 239-260.

Frankel, Jeffrey and K. Froot, 1989, Forward discount bias: Is it an exchange risk premium?, Quarterly Journal of Economics 1, 139-161.

Gallant, A. Ronald, 1987, Nonlinear statistical models (Wiley, New York, NY).

Gallant, A. Ronald and Geraldo Souza, 1991, On the asymptotic normality of Fourier flexible form estimates, Journal of Econometrics 50, 329-353.

Gallant, A. Ronald and George Tauchen, 1989, Seminonparametric estimation of conditionally constrained heterogeneous processes: Asset pricing applications, Econometrica 57, 1091-1120.

Gallant, A. Ronald and George Tauchen, 1992a, A nonparametric approach to nonlinear time series analysis: Estimation and simulation, in: David Brillinger, Peter Caines, John Geweke, Emanuel Parzen, Murray Rosenblatt, and Murad S. Taqqu, eds., New directions in time series analysis, Part II (Springer-Verlag, New York, NY) 71-92.

Gallant, A. Ronald and George Tauchen, 1992b, Which moments to match?, Manuscript (Department of Economics, Duke University, Durham, NC).

Gallant, A. Ronald, Lars Peter Hansen, and George Tauchen, 1990, Using conditional moments of asset payoffs to infer the volatility of intertemporal marginal rates of substitution, Journal of Econometrics $45,141-179$.

Gallant, A. Ronald, David Hsieh, and George Tauchen, 1991, On fitting a recalcitrant series: The pound/dollar exchange rate, 1974-83, in: William A. Barnett, James Powell, and George E. Tauchen, eds., Nonparametric and semiparametric methods in econometrics and statistics. Proceedings of the fifth international symposium in economic theory and econometrics (Cambridge University Press, Cambridge) 199-240.

Gourieroux, C., A. Monfort, and E. Renault, 1992, Indirect inference, Manuscript (CRESTCEPREMAP, Paris).

Hannan, E.J., 1987, Rational transfer function approximation, Statistical Science 2, 1029-1054.

Hansen, Lars Peter, 1982, Large sample properties of generalized methods of moments estimators, Econometrica 50, 1029-1054.

Hansen, Lars Peter and Robert Hodrick, 1983, Risk averse speculation in the forward foreign exchange market: An econometric analysis, in: Jacob Frenkel, ed., Exchange rates and international macroeconomics (University of Chicago Press, Chicago, IL) 113-152.

Hansen, Lars Peter and Ravi Jagannathan, 1991, Implications of security market data for models of dynamic economies, Journal of Political Economy 99, 225-262.

Hansen, Lars Peter and Kenneth J. Singleton, 1982. Generalized instrumental variables estimators of nonlinear rational expectations models. Econometrica 50, 1269-1286. 
Heaton, John, 1993, The interaction between time-nonseparable preferences and temporal aggregation, Econometrica 61, 353-386.

Hodrick, Robert, 1989, Risk, uncertainty, and exchange rates, Journal of Monetary Economics 23, 433-459.

Hodrick, Robert and S. Srivastava, 1986, The covariation of risk premiums and expected future spot exchange rates, Journal of International Money and Finance 5, 5-22.

Hodrick, Robert, Narayana Kocherlakota, and Deborah Lucas, 1991, The variability of velocity in cash-in-advance models, Journal of Political Economy 99, 358-384.

Hsieh, David, 1989, Testing for nonlinear dependence in daily foreign exchange rates, Journal of Business 62, 339-368.

Huang, R.D., 1981, The monetary approach to exchange rates in an efficient foreign exchange market, Journal of Finance $36,31-41$.

Ingram, Beth F. and B.S. Lee, 1991, Simulation estimation of time series models, Journal of Econometrics 47, 197-205.

Judd, Kenneth L., Minimum weighted least residual methods for solving aggregate growth models, Manuscript (Federal Reserve Bank of Minneapolis, Minneapolis, MN).

Lucas, Robert E., Jr., 1982, Interest rates and currency prices in a two-country world, Journal of Monetary Economics 10, 335-360.

Manski, Charles F., 1983, Closest empirical distribution estimation, Econometrica 51, 305-320.

Marcet, Albert, 1991, Solution of nonlinear models by parameterizing expectations: An application to asset pricing with production, Manuscript (Department of Economics, Carnegie Mellon University, Pittsburgh, PA).

Marshall, David, 1988, Inflation and asset returns in a monetary economy with transactions costs, Manuscript (Department of Economics, Northwestern University, Evanston, IL).

Matsuyama, Kiminori, 1990, Sunspot equilibria (rational bubbles) in a model of money-in-the-utilityfunction, Journal of Monetary Economics 25, 127-144.

Meese, R. and Kenneth Rogoff, 1983a, Empirical exchange rate models of the seventies: Do they fit out of sample?, Journal of International Economics 14, 3-24.

Meese, R. and Kenneth Rogoff, 1983b, The out-of-sample failure of empirical exchange rate models: Sampling error or misspecification?, in: Jacob Frenkel, ed., Exchange rates and international macroeconomics (University of Chicago Press, Chicago, IL) 67-112.

Nelson, Daniel, 1991, Conditional heteroskedasticity in asset returns: A new approach, Econometrica $59,347-370$.

Park. Woo, 1985, Money in the utility function via shopping time technology: An empirical study, Manuscript (Department of Economics, Carnegie Mellon University, Pittsburgh, PA).

Potscher, Benedikt M., 1989, Model selection under nonstationarity: Autoregressive models and stochastic linear models. Annals of Statistics 17. 347-370.

Schwarz, Gideon, 1978, Estimating the dimension of a model, Annals of Statistics 6, 461-464.

Singleton, Kenneth J., 1987, Speculation and volatility of foreign currency exchange rates, CarnegieRochester Conference Series on Public Policy 26, 9-56.

Smith, Anthony A., 1990, Three essays on the solution and estimation of dynamic macroeconomic models, Ph.D. dissertation (Duke University, Durham, NC).

Smith, Anthony A.. 1992, Estimating nonlinear time series models using simulated vector autoregressions: Two approaches, Manuscript (Queen's University, Kingston, Ont.).

Stigum. Marcia, 1990, The money market, 3rd ed. (Dow Jones-Irwin, Homewood, IL).

Stockman. Alan C., 1980, Theory of exchange rate determination, Journal of Political Economy 88, 673-698.

Sundaresan, Suresh, 1989. Intertemporally dependent preferences and the volatility of consumption and wealth, Review of Financial Studies 2, 73-89.

Svensson, Lars E.O., 1985, Currency prices, terms of trade, and interest rates: A general equilibrium asset-pricing cash-in-advance approach, Journal of International Economics 18, 17-41. 
Tauchen, George, 1990, Associate editor's introduction to the special section on solving nonlinear rational expectations models, Journal of Business and Economic Statistics 8, 1-1.

Tauchen, George and Robert Hussey, 1991, Quadrature-based methods for obtaining approximate solutions to nonlinear asset pricing models. Econometrica 59, 371-396.

Taylor, John B. and Harald Uhlig, 1990, Solving nonlinear stochastic growth models: A comparison of alternative solution methods. Journal of Business and Economic Statistics 8, 1-17.

Tobin, James, 1978, A proposal for international monetary reform, Eastern Economic Journal 4. 153159. 